XXIX.-Semi-regular Networks of the Plane in Absolute Geometry. By Duncan M. Y. Sommerville, M.A., B.Sc., University of St Andrews. Communicated b.y Professor P. R. Scott Lanc. (With Twelve Plates.)

(The cost of the Illustrations of this Paper was defrayed by the CAINEA;IE. TRIST.)

(Read December 19, 1904. Issued separately Augrust :3n, 19615.)

$\$ 1$. The networks considered in the following paper are those networks of the plane whose meshes are regular polygons with the same length of side.

When the polygons are all of the same kind the network is called regulur, otherwise it is semi-regular.

The regular networks have been investigated for the three geometries from various standpoints, the chief of which may be noted.

1. The three geometries can be treated separately. For Euclidean geometry we have then to find what regular polygons will exactly fill up the space round a point. For elliptic geometry we have to find the regular divisions of the sphere, or, what is the same thing, the regular polyhedra in ordinary space. The regular networks which do not belong to either of these classes are then those of the hyperbolic plane.

2. The problem is identical with that of finding the partitions of a polygon into polygons of the same kind, with the same number of polygons at each point. ${ }^{*}$ The boundary polygon is one of the meshes of the network. For elliptic networks the boundary is finite, for Euclidean networks it is wholly at infinity, and for hyperbolic networks it is wholly ideal.

This method gives a convenient mode of representing the networks, viz., by their stereographic projections upon the Euclidean plane. This representation will be employed throughout.

3. The problem corresponds to a particular case of the problem of determining all discontinuous groups of motions in the plane. ${ }^{+}$

It will be convenient here to collect the results. If $n$ is the number of sides of each polygon, $p$ the number of lines or polygons meeting at each point, $\mathrm{N}_{2}, \mathrm{~N}_{1}, \mathrm{~N}_{0}$ the number of meshes, lines, and nodes respectively, the results may be summariser as follows :-

1. On the Elliptic plane there are five regular networks, corresponding to the five 1883.

* See V. Schlegel, "Theorie der homogenen zusamnengesetzten Raumgebilde," Nova Acta, Bd. xliv., Nr. 4,

+ W. Dyck, "Gruppentheoretische Studien," Math. Annalen, xx. 1-4t (1882), and W. Bornside, "Theory of Groups," ch. xii., xiii. Also Kletin and Fricke, "Theorie der elliptischen Modulfunetionen." (For these references I am indebted to the referee.)

TRANS. ROY. SOC. EDIN., VOL. XLI. PART III. (NO. ə9). 
resular polyhedra. They are arranged in conjugate pairs, the number of meshes in one being equal to the number of nodes in the conjugate network. One is selfconjugate.

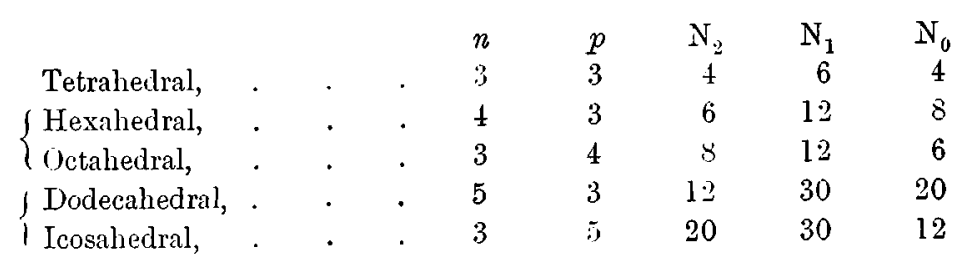

๖. On the Euclidean plane there are three regular networks, all infinite.

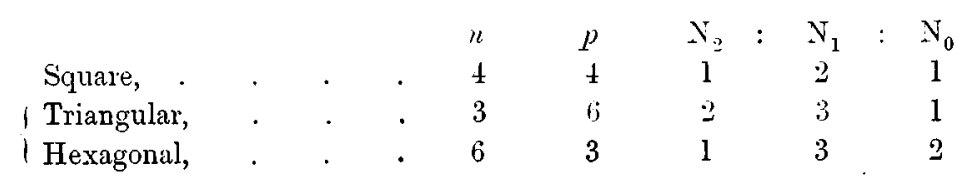

3. On the $\mathrm{H}_{y}$ perbolic plane there are an infinite number, all infinite.

$$
\begin{aligned}
& p=3,4,5,6,>6 \\
& n>6,4,3,3, \text { any value } \\
& \mathrm{N}_{2}: \mathrm{N}_{1}: \mathrm{N}_{0}=2 p: n p: 2 n
\end{aligned}
$$

$\$ 2$. We proceed to investigate the semi-regular networks, and we shall take the three geometries separately.

\section{The Euclidean Plane.}

We shall consider, first, how the space about a point can be exactly filled with regular polygons. Each combination of polygons satisfying this condition determines a species of node, and all the semi-regular networks must be built up out of the various possible species of nodes. Two networks will be consiclered to be of the same type when they contain only nodes of the same species. It is obvious that there may be varieties of the same type. The types will be rivided into Groups according to the kinds of polygons involved, and the groups into Classes according to the number of kinds of polygons. Class A. consists of the regular networks, and contains three groups with one unvaried type in each. The simplest type in any group is that which contains only one species of node. I call this the simple t!lpe; other types I call composite. A group does not necessarily contain the simple type.

\section{The Species of Norles.}

$\$ 3$. The angle of at regular $u$-gon is given by the formula

$$
\mu_{n}=\left(1-\frac{2}{n}\right) 180^{\circ}
$$

The following table of values of $a_{n}$ will be immediately useful : 


\begin{tabular}{|c|c|c|c|c|c|c|c}
\hline$n$ & 3 & 4 & 5 & 6 & 7 & 8 & 12 \\
\hline$a_{n}$ & 60 & 90 & 108 & 120 & 124 & 135 & 150 \\
\hline
\end{tabular}

T'aking the four simplest polygons, we find that the sum of the angles is $378^{\circ}$, i. $>5,50^{\circ}$. Hence there cannot be more then three different linds of jolygons at a print. The species of nodes therefore fall under three classis.

Class A. contains the homogeneous nodes. Denoting the rogular polygons by their initial letters, the nodes of Class $\mathrm{A}$. can be denoted by
1. $\mathrm{T}_{6}$.
2. $S_{4}$.
3. $\mathrm{H}_{3}$.

$\$$ 4. Class B. Let there be at a point $p_{1} n_{1}$-gons and $p_{2} n_{-}$-gons. Then we have

hence from (1)

$$
p_{1} a_{1}+p_{2} a_{2}=2 \pi,
$$

$$
p_{1}\left(1-\frac{2}{n_{1}}\right)+p_{2}\left(1-\frac{2}{n_{2}}\right)=2 .
$$

Integralising, we obtain on the left the function

$$
n_{1} n_{2}(2-p)+2\left(p_{1} n_{2}+p_{2} n_{1}\right)
$$

where $p=p_{1}+p_{2}$. We shall denote this by $\Delta$. It is easily seen that the sign of $\Delta$ characterises the network as elliptic, hyperbolic, or Euclidean. For Euclidean networks $\Delta$ is always zero; for elliptic and hyperbolic networks $\Delta \gtrless 0$ respectively.

For the regular networks there is a corresponding function $u(2-p)+2 p$, and for three kinds of polygons we shall find a similar function. Where there is no risk of confusion we shall call each of these $\Delta$. The values of $\Delta$ for the regular networks are as follows: Tetrahedral 3, Hexahedral and Octahedral 2, Dodecahedral and Icosahedral 1.

Solving now for $n_{2}$, we have

$$
n_{2}=\frac{2 l_{1} n_{1}}{\left.n_{1}(p-2)-2 p_{1}\right)} .
$$

We have to find the integral solutions of this equation under the following ammlitions: $u_{1} \neq n_{2}>3, p_{1}, p_{2}>0, p \nless 3$ and $p \ngtr 5$, therefore $p_{1}, p_{0} \neq 4$.

The only possible sets of values of $p_{1}, p_{2}$ are then

$$
\begin{aligned}
& \mu_{1}=1,1,1,2,2 . \\
& \mu_{2}=2,3,4,2.3 .
\end{aligned}
$$

We shall take these cases in succession.

$$
\begin{aligned}
& \left.\begin{array}{l}
p_{1}=1 \\
p_{2}=2
\end{array}\right\} \\
& \text { whence } n_{1}=3,4,10 \text {, } \\
& n_{2}=12,8 \text {, } \therefore \text {. } \\
& \left.\begin{array}{l}
p_{1}=1 \\
p_{2}=3
\end{array}\right\} \quad n_{2}=\frac{3 n_{1}}{n_{1}-1}=3+\bar{n}_{1}^{3}-1 .
\end{aligned}
$$


There are no unequal values of $n_{1}$ and $n_{2}$ satisfying this equation.

$$
\begin{aligned}
& \left.\begin{array}{l}
p_{1}=1 \\
p_{2}=4
\end{array}\right\} \begin{array}{l}
n_{2}=\frac{8 n_{1}}{3 n_{1}}-2 \text { or } 3 n_{2}=8+\frac{16}{3 n_{1}-2} \\
\text { whence } \begin{array}{l}
n_{1}=6 \\
n_{2}=3 .
\end{array}
\end{array} \\
& \begin{array}{ll}
\left.\mu_{1}=2\right\} & n_{2}=\frac{2 n_{1}}{n_{1}-2}=2+\frac{4}{n_{1}-2} \\
\left.\mu_{2}=2\right\} & n_{1}=3
\end{array} \\
& \text { whence } \begin{array}{l}
n_{1}=3 \\
n_{2}=6
\end{array} \\
& \left.\begin{array}{l}
\nu_{1}=2 \\
\nu_{2}=3
\end{array}\right\} \begin{array}{l}
n_{2}=\frac{6 n_{1}}{3 n_{1}-4}=2+\frac{8}{3 n_{1}-4} \\
\text { whence } \begin{array}{l}
n_{1}=4 \\
n_{2}=3
\end{array}
\end{array}
\end{aligned}
$$

There are thus six speries of nodes in this class. They may be denoted by $\mathrm{TD}_{2}$, $\mathrm{SO}_{3}, \operatorname{Dec} \mathrm{P}_{2}, \mathrm{~T}_{4} \mathrm{H}, \mathrm{T}_{2} \mathrm{H}_{2}, \mathrm{~T}_{3} \mathrm{~S}_{2}$.

§. Clasis C. Here we have

$$
\begin{gathered}
p_{1}\left(1-\frac{2}{n_{1}}\right)+p_{2}\left(1-\frac{2}{n_{2}}\right)+p_{3}\left(1-\frac{2}{n_{3}}\right)=2 \\
\text { or } \quad د \equiv n_{1} n_{2} n_{3}(2-\nu)+2\left(p_{1} n_{2} n_{3}+p_{2} n_{3} n_{1}+p_{3} n_{1} n_{2}\right)=0 . \\
\text { Solving for } n_{3}, \quad n_{3}=\frac{2 p_{3} n_{1} n_{2}}{n_{2}\left(p-2 \cdot n_{1}-2 p_{1}\right)-2 p_{2} n_{1}} .
\end{gathered}
$$

We have to solve this equation in integers under the following conditions : $n_{1} \neq n_{2} \neq n_{:}>3$, $p_{1}, p_{2}, p_{3}>0, p \nless 3$, also $p \ngtr 4$ (for $3 \alpha_{3}+\alpha_{4}+\alpha_{5}>360^{\circ}$ ), therefore $p_{1}, p_{2}, p_{3} \ngtr 2$. Further, if $p_{1}=2, n_{1}$ must be either 3 or 4 , for $2 a_{3}+a_{3}+a_{4}>360^{\circ}$. Again, we cannot have $n_{1}=5, n_{2}=6, n_{3}=7$ together, for $\alpha_{5}+\alpha_{6}+\alpha_{7}>360^{\circ}$.

The following are therefore the only possible sets of values of $p_{1}, p_{2}, p_{3}, u_{1}$ :

$$
p_{1}=1, \text { or } 2 ; p_{2}=p_{3}=1 ; n_{1}=3, \text { or } 4 \text {. }
$$

We shall take these cases in succession.

$$
\begin{aligned}
& \left.\begin{array}{rl}
y_{1}=p_{2}= & p_{3}=1 \\
n_{1}=3
\end{array}\right\} \quad n_{3}=\frac{6 n_{2}}{n_{2}-6}=6+\frac{36}{n_{2}-6} \\
& \text { whence } n_{2}=7,8,9,10 \text {, } \\
& n_{3}=42,24,18,15 \\
& \left.l^{\prime \prime}=p_{2}=\begin{array}{l}
p_{3}=1 \\
n_{1}=4
\end{array}\right\} \quad n_{3}=\frac{4 n_{2}}{n_{2}-4}=4+\frac{16}{n_{2}-4} \\
& \text { whence } n_{2}=5,6 \text {, }
\end{aligned}
$$

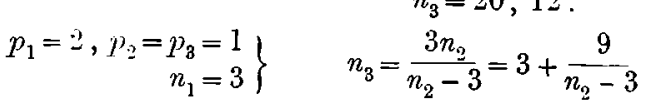

$$
\begin{aligned}
& \text { whence } n_{2}=4 \text {, }
\end{aligned}
$$

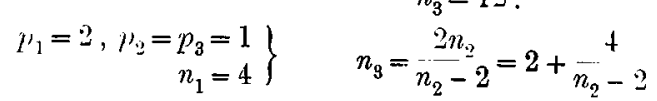

$$
\begin{aligned}
& \text { whence } \begin{aligned}
n_{2} & =3, \\
n_{2} & =6 .
\end{aligned}
\end{aligned}
$$

There are thus eight species of nodes in this class. 
§. Collecting all the species of nodes, we can arrange them in the following scheme :

$$
\begin{aligned}
& \text { Class A. 1. } T_{6} \text {. } \\
& \text { Class B. 4, } \mathrm{T}_{3} \mathrm{~S}_{2} \text {. } \\
& \text { Class C. 10. 'Tr...II. } \\
& \text { 2. } s_{1} \text {. } \\
& \text { 3. } \mathrm{II}_{3} \text {. } \\
& \text { 5. } \mathrm{T}_{2} \mathrm{I}_{2} \text {. } \quad \text { i. } \mathrm{T}_{4} \mathrm{H} \\
& \text { 11. TSI). 12. SHD } \\
& \text { 7. } \mathrm{TD}_{2} . \quad 8 . \mathrm{SO}_{2} \text {. } \\
& \begin{array}{rrrrrrr}
{[13-17 .]} & n_{1} & 4, & 3, & 3, & 3, & 3, \\
& n_{2} & 5, & 10, & 9, & 8, & 7, \\
& n_{3} & 20, & 15, & 18, & 24, & 42 .
\end{array}
\end{aligned}
$$$$
\text { [9.] } \mathrm{P}_{2} \text { Dec. }
$$

In future we shall refer to these nodes by their numbers in this scheme.

Of the seventeen species of nodes, only eleven are capable of development to form networks.

In No. 9 the pentagon must be surrounded alternately with pentagons and decagons, which is impossible since 5 is odd.

In the same way, when $p_{1}=p_{2}=p_{3}=1, n_{1}, n_{2}$ and $n_{3}$ must all be even, for the $n_{1}$-gon must be surrounded alternately with $n_{2}$-gons and $n_{3}$-gons. Hence 13-17 cannot be developed by themselves; nor can they be developed in combination, for each contains a polygon which is not contained in any other node. Similarly, 9 cannot be developed in combination, hence these six species are excluded from all the networks.

Again, $\mathrm{T}_{2} \mathrm{SD}$ cannot be developed by itself, for, taking the square (fig. 1, Plate IV.), we must have a dodecagon on one side and on the adjacent sides double triangles. At the free corners of the square we must now have dorlecagons, but this brings two dodecagons at a point and introduces 7 ; excluding this, we must introduce 4 .

$\S 7$. We can now divide the types of networks into groups and classes. Five kinds of polygons are at our disposal, but octagons only occur in the combination $\mathrm{SO}_{2}$, hence there are only four classes.

Class A. Regular networks.

Group I. Triangles (1).

$"$ II. Squares (2).

, III. Hexagons (3).

Class B. Two kinds of polygons.

Group I. Triangles and Squares $(1,2,4)$.

II. Triangles and Hexagons $(1,3,5,6)$.

" III. Triangles and Dodecagons $(1,7)$.

, IV. Squares and Octagons $(2,8)$.

Class C. 'Three kinds of polygons.

Group I. Triangles, Squares, and Hexagons $(1,2,3,4,5,6,10)$.

$" \quad$ II. Triangles, Squ 1 res, and Dodecagons $(1,2,4,7,11)$.

"III. Squares, Hexagons, and Dodecagons $(2,3,12)$. 
Class D. Four kinds of polygons.

Group. I. Triangles, Squares, Hexagons, and Dodecagons $(1,2,3,4,5,6,7$, $10,11,12)$.

The numbers within the brackets denote the species of nodes which the group admits.

$$
\text { The simple Types. }
$$

§ 8. Now let us consider the simple types. I observe, in the first plice, that when the species of node admits of no variation, the simple type is in general unvaried.

The unique nodes are the following:

$$
\begin{aligned}
& \text { 1․ Class A, } \\
& \text { ¿.. Those in which } \eta^{\prime}=3, \\
& \text { 3. } p_{1}=1, p^{\prime 2}=4,
\end{aligned}
$$

while the following are varied:

$$
\begin{aligned}
& 1^{\prime \prime} \cdot p_{1}=p_{2}=2 . \quad \text { Two forms, } \mathrm{M}_{2} \mathrm{~N}_{2} \text { and }(\mathrm{MN})_{2} . \\
& 2^{\circ} . p_{1}=p_{2}=1, p_{3}=2 . \text { Two forms, LMN } \mathrm{MN}_{2} \text { and LNIIN }
\end{aligned}
$$

We have then the unique simple types.

$$
\begin{aligned}
& \text { Class A. } \mathrm{T}_{6}, \mathrm{~S}_{4}, \mathrm{H}_{3} . \\
& \text {, B. } \mathrm{T}_{4} \mathrm{H}, \mathrm{TD}_{2}, \mathrm{SO}_{2} \text { (figs. } 4,2,3 \text { ). } \\
& \text {, C. } \mathrm{SHD} \text { (fig. 5). }
\end{aligned}
$$

The type $\mathrm{T}_{4} \mathrm{H}$ is one exception to the rule stated above, for it does admit of a variation. The network is asymmetrical, its mirror image being different from itself. It exists. therefore, in two enantiomorphic forms. The one can be obtained from the other by turning the plane over.

Of the other groups, $\therefore$ II. and D. I. do not possess simple types, and there remain the three simple types $\mathrm{T}_{3} \mathrm{~S}_{2}, \mathrm{~T}_{2} \mathrm{H}_{2}$, and $\mathrm{TS}_{2} \mathrm{H}$, each of which is apable of infinite variation.

$\mathrm{T}_{2} \mathrm{H}_{2}$ has a variety in which there are no two triangles and no two hexagons together. We shall call- this the fiumlamental variety. The opposite sides of any hexagon, when produced, define a strip which is capable of displacement without affecting the rest of the network. All the varieties can then be obtilined by displacing any number of such parallel strips through a distance equal to the length of the side (fig. 6).

$\mathrm{TS}_{\varepsilon} \mathrm{H}$ has the fundamental variety in which there are no two squares together. Each hexagon is surrounded by squares and triangles, forming a group whose boundary is a regular dodecagon. All the varieties can then be obtained by turning any number of such groups through $\frac{\pi}{6}$. This operation, performerl upon a single group, brings two squares together; performed upon two adjacent but not overlapping groups, it brings three squares together (fig. 7). 
§. The unique types and the fundamental varieties of $\mathrm{T}_{2} \mathrm{H}_{2}$ and $\mathrm{TS} \cdot \mathrm{H}$ can be obtained from the regular networks by fairly olvious dissections. Thus, SHD (fig. 5) is obtained from either the triangulir or the hexiggonal network; for the squares, hexagons, and denlemuns have $(1,1)$ correspomlenes with the lines, meshes, and nodes respectively of the trimgular network. In a similar way $\mathrm{T}_{4} \mathrm{H}$ (fig. 4) is obtained from the sime metwork; to cach mesh there corresponds a triangle, to each node a hexagon, and to each line two triangles. And $\cdots$ for the others: it is only necessary to compare the figures, given below $(\S 11)$, which represent the relative numbers of the various polygons, with the numbers of nodes, lines, and meshes in the regular networks. In the diagrams given for the unique types the regular network is indicated by shading.

$\mathrm{T}_{4} \mathrm{H}$ and all the varieties of $\mathrm{T}_{2} \mathrm{H}_{2}$ can also be obtained from the rezular triangular network by replacing all the groups of six covertical triangles by hexagons; and $\mathrm{TS}_{2} \mathrm{H}$ can be obtained from SHD by bordering every dodecagon internally with squares and triangles.

$\$ 10$. The type $\mathrm{T}_{3}, \mathrm{~s}$ forms an exception to what has been said regarding the way in which the network may be obtained. One of its varieties, that in which no two squares are together, can be obtained in a simple way from the regular square network; to each mesh corresponds a rifuare, to each node a square, and to each line two triangles. But the other varieties cannot be obtained from this, nor, in general, in any simple way from the square network. The following forms may be enumerated, though the list is not exhaustive :--

(1) $1,2,3, \ldots$ squares always together. Each of these is unique, and the series forms a general type of variety, admitting of an infinite number of mixtures (tig. 8).

$(\because)), 3, \ldots$ squares or fewer together. Here we can distinguish

(i.) Two similar types, in which there occurs once only (a) a single triangle surrounded by three squares, $(b)$ a triangular group of four triangles surrounded by three double squares. The network raliates from this figure as centre (fig. 10).

(ii.) A general type, obtainable by a dissection of the square network, in which (1) and (b) are excluded (fig. 9).

(iii.) Further, if an unlimited number of squares may be together, the groups $(\alpha)$ aml (b) may occur more than once, or together.

11. From what has been said it is evident that for any of the simple types, with the possible exception of $\mathrm{T}_{3} \mathrm{~S}_{\mathrm{L}}$, the relative number of the several kinds of polygons is definite, and the same for all the varieties. These numbers can be found by inspection and a knowledge of the number of meshes, lines, and nodes in the regular networks. General expressions for the ratios may be found as follows. The results show that $\mathrm{T}_{\mathrm{i}} \mathrm{S}_{\text {: }}$ is not an exception in this respect. 
Let $p^{\prime}, p^{\prime \prime}, p^{\prime \prime \prime}$ be the number of $n^{\prime}-, n^{\prime \prime}-, n^{\prime \prime \prime}$-gons meeting at each point; $\mathrm{N}_{0}$ the number of nodes, $\mathrm{N}_{2}{ }^{\prime}, \mathrm{N}_{2}^{\prime \prime}, \mathrm{N}_{2}^{\prime \prime \prime}$ the number of $n^{\prime}-, n^{\prime \prime}-, n^{\prime \prime \prime}$-gons in the whole network.

Then each $n^{\prime}$-gon has $n^{\prime}$ angles; but if we count up the whole number of angles contributed by all the $n^{\prime}$-gons, each is counted $p^{\prime}$ times.

$$
\begin{aligned}
\text { Hence } & n^{\prime} \mathrm{N}_{2}^{\prime}=y^{\prime} \mathrm{N}_{0}, \\
n^{\prime \prime} \mathrm{N}_{2}^{\prime \prime} & =p^{\prime \prime} \mathrm{N}_{0}, \\
n^{\prime \prime \prime} \mathrm{N}_{2}^{\prime \prime \prime} & =p^{\prime \prime \prime} \mathrm{N}_{0} . \\
\text { Thimilarly } & p^{\prime}: \frac{p^{\prime \prime}}{n^{\prime \prime}}: \frac{p^{\prime \prime \prime}}{n^{\prime \prime \prime}} .
\end{aligned}
$$

So that the relative number of polygons is the same for all varieties of the same type.

$$
\text { For } \begin{aligned}
\mathrm{T}_{3} \mathrm{~s}_{2}, & \mathrm{~T}: \mathrm{S}=2: 1 \\
\mathrm{~T}_{2} \mathrm{H}_{2}, & \mathrm{~T}: \mathrm{H}:=2: 1 \\
\mathrm{~T}_{4} \mathrm{H}, & \mathrm{T}: \mathrm{H}=8: 1 \\
\mathrm{TI} \mathrm{I}_{2}, & \mathrm{~T}: \mathrm{D}=2: 1
\end{aligned}
$$

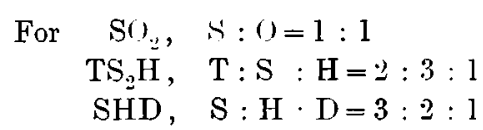

$\S 12$. Let us investigate the analogous formulæ for composite types. Let $_{1} \mathrm{~N}_{0}$ be the number of nodes at which there are $p_{1}^{\prime} n^{\prime}$-gons, $p_{1}^{\prime \prime} n^{\prime \prime}$-gons, $p_{1}^{\prime \prime \prime} n^{\prime \prime \prime}$-gons, and $p_{1}{ }^{\mathrm{iv}} n^{\mathrm{iv}}$-gons, where one at least of the quantities $p_{1}$ is zero, and let

$$
\text { Then } \quad \begin{aligned}
&{ }_{1} \mathrm{~N}_{0} \cdot{ }_{2} \mathrm{~N}_{0} \ldots \ldots, \mathrm{N}_{0}=1: k_{1} \ldots \ldots: k_{r-1} . \\
& n^{\prime} \mathrm{N}_{2}{ }_{2}=p_{1}{ }_{1} \mathrm{~N}_{0}+{ }^{\prime},{ }_{2}^{\prime} \mathrm{N}_{0}+\ldots+p_{r}^{\prime}, \mathrm{N}_{0} \\
&=\left(p_{1}^{\prime}+h_{1} p_{2}^{\prime}+\ldots+k_{r-1} p_{r}^{\prime}\right)_{1} \mathrm{~N}_{0}
\end{aligned}
$$

Let $\mathrm{N}_{2}, \mathrm{~N}_{1}, \mathrm{~N}_{0}$ have their usual meanings, then

$$
\begin{aligned}
& \mathrm{N}_{0}={ }_{1} \mathrm{~N}_{0}+\ldots+{ }_{r} \mathrm{~N}_{0}=\left(1+k_{1}+\ldots+i_{i,-1}\right)_{1} \mathrm{~N}_{0} \\
& \mathrm{~N}_{2}=\mathrm{N}_{2}^{\prime}+\mathrm{N}_{2}^{\prime \prime}+\mathrm{N}_{2}^{\prime \prime \prime}+\mathrm{N}_{2}{ }^{\text {iv }} .
\end{aligned}
$$

Also, by the analogue of EULER's polyhedral formula,

$$
\mathrm{N}_{2}-\mathrm{N}_{1}+\mathrm{N}_{0}=1 \text {. }
$$

$\mathrm{N}_{1}$ can also be expressed in terms of ${ }_{1} \mathrm{~N}_{0}, \ldots,{ }_{r} \mathrm{~N}_{0}$ thus : at each of the ${ }_{1} \mathrm{~N}_{0}$ points there are $p_{1}$ lines, and each line joins two points, hence

whence we get

$$
2 \mathrm{~N}_{1}=p_{11} \mathrm{~N}_{0}+\ldots+p_{r} \mathrm{~N}_{0},
$$

$$
\sum_{i=1}^{i=4}\left(\frac{1}{n^{i}} \sum_{\lambda=1}^{\lambda=r} k_{\lambda-1} p_{\lambda}^{i}\right)-\frac{1}{2} \sum_{\lambda=1}^{\lambda=r} k_{\lambda-1} p_{\lambda}+\sum_{\lambda=1}^{\lambda=r} k_{\lambda-1}=\frac{1}{1 N_{0}} .
$$

Now, since the number of species of nodes in the whole network is finite, one at least of the quantities ${ }_{1} \mathrm{~N}_{0}, \ldots,{ }_{r} \mathrm{~N}_{0}$ must be infinite. Hence we may put ${ }_{1} \mathrm{~N}_{0}=\infty$. The equation then becomes

$$
\sum_{\lambda=1}^{\lambda=r}\left(\frac{p_{\lambda}^{\prime}}{n^{\prime}}+\frac{p_{\lambda}^{\prime \prime}}{n^{\prime \prime}}+\frac{p_{\lambda}^{\prime \prime \prime}}{n^{\prime \prime \prime}}+\frac{p_{\lambda}{ }^{\mathrm{iv}}}{n^{\mathrm{iv}}}-\frac{p_{\lambda}}{2}+1\right) k_{\lambda-1}=0 .
$$

But this is an identity on account of the fundamental relation $\Delta=0$. Without further 
conditions, then, we cannot find any relation between the $k$ 's, which are therefore indeterminate. This shows that in general a composite type admits of infinite variation, the ratios of the number of polygons being indefinite.

\section{Composite Typess.}

$\S 13$. We have defined two networks to be of the same type when they involve only nodes of the sime species, whatever may be their form and structure. The determination of the composite types in any group thus reduces to finding all the possible combinations of nodes admitted by the group. In order to eliminate the impossible combinations, we can find what combinations must occur. We shall take each group in turn.

Class B. I. Triangles and Squares $(1,2,4)$.

(a) 2 must be accompanied by 4 (fig. 11).

Hence the only combinations are 1,$4 ; 2,4 ; 1,2,4$, each of which gives a composite type. Examples of each are given by joining together infinite parallel strips of the triangular and square networks.

II. Triangles and Hexagons $(1,3,5,6)$.

(b) 3 must be accompanied by 5 (fig. 12).

Hence the only combinations are 1,$5 ; 1,6 ; 3,5 ; 5,6 ; 1,3,5 ; 1,5,6$; $3,5,6 ; 1,3,5,6$. There are thus eight composite types. Every variety of them can be obtained from the triangular network by replacing groups of six triangles by hexagons.

III. Triangles and Dodecagons $(1,7)$.

IV. Squares and Octagons $(2,8)$.

Each of these is unique and admits of no composite types.

$\S$ 14. Class C. I. Triangles, Squares, and Hexagons $(1,2,3,4,5,6,10)$.

The number of combinations of these numbers, two or more together, so as to include the three polygons, is 105 . We proceed to establish rules for the rejection of impossible combinations.

(a) 2 must be accompanied by 4 or 10 (fig. 11).

(b) 3 " , , 5 ; and either 6, or 10 and 4 (fig. 12).

Exclude 6 ; then we must have 10 , since squares and hexagons come together; and since there are always two triangles together, we must also have 4 .

Exclude 10; then squares cannot be introduced till the hexagons have been surrounded by triangles, and we have 6 .

(c) 5 must be accompanied by either 10, or 4 and 6 (fig. 12).

Exclude 10; before squares can be arded we get 6 , and the further addition of squares gives 4 .

TRANS. ROY. SOC. EDIN., VOL. XLI. PART. III. (NO. 29). 
Hence if 10 is excluded, we must have 4 and 6 .

For 4 is necessary in order to give squares, and if we exclude 6 we also exclude 5 , and therefore 3 . We are then left with only $1,2,4$, which do not involve hexagons.

(d) 6 must be accompanied by 4 or 5 .

Excluding 4 and 5, the hexagon must be surrounded by triangles, and squares can never be introduced without producing 4 .

(c) 1 must be accompanied by either 4 , or 5 and 6 .

Exclude 5 and 6 ; then $\mathrm{T}_{6}$ must be surrounded by either triangles, or triangles and squares. Since hexagons are excluded at this stage, either of these introduces 4.

Exclude 4 and 6 ; then $\mathrm{T}_{6}$ must be surrounded by hexagons. Now squares can only be introduced after the concavities have been filled up. If we fill them with hexagons fresh gaps are produced, and if we fill them with triangles there are always two triangles together, and the addition of squares is impossible without giving 4 . Hence we cannot exclude both 4 and 6 .

Excluile 4 and 5 ; then 6 is also excluded by $(d)$, and $\mathrm{T}_{6}$ can only be surrounded by triangles.

Also 1 can only be continued by 4,5 , or 6 ; hence if we exclude 4 , we must have both 5 and 6 .

Rejecting according to these rules, we are left with forty-seven combinations, each of which gives a composite type. The combinations may be represented by the following notation. Let $\mathrm{C}_{r}\left(a_{1}, \ldots, a_{n}\right)$ stand for any one combination of $r$ or more of the $\alpha$ s, then the forty-seven combinations are

$$
\begin{gathered}
5+C_{2}(4,6,10)+C_{0}(1,2,3) \\
++C_{1}(6,10)+C_{0}(1,2) \\
10+C_{1}(2,5)
\end{gathered}
$$

$\$$ 15. Class C. II. Triangles, Squares, and Dodecagons $(1,2,4,7,11)$.

(a) 2 must be accompanied by 4 (fig. 11).

$(f) 7 \quad, \quad, \quad, \quad 11$ (fig. 1 ).

After rejection there are left eleven combinations, all of which give composite types except 1, 7, 11 . There are therefore the following ten composite types in this group:

$$
4,11+\mathrm{C}_{0}(1,2,7) ; \quad 1,11 ; \quad 7,11 .
$$

III. Squares, Hexagons, and Dorlemigons (2, 3, 12).

Exclurling triangles, 12 rin only he continued in one way, hence there are no composite types in this group.

§ 16. Class D. Triangles, Squares, Hexagons, and Dodecagons $(1,2,3,4,5,6,7$, $10,11,12)$. 
The number of combinations of two or more together of these numbers which involve all four polygons is 856 , but we have the following rules for the rejection of impossible combinations:

(a) 2 must be accompanied by 4 or 10 .

(b) $3 \quad, \quad, \quad, \quad 5$; amd rither $6, \ldots 10$ and 4 .

(c) $5 \quad, \quad, \quad, \quad$ either 10 , or 4 amd is.

(d) $6 \quad, \quad, \quad, \quad 4$ 川 5 .

$(f) 7 \quad, \quad, \quad, \quad, \quad 11$.

The previous proofs of these still hold.

(e) 1 must be ancompanied by either 4, 5 and 6 , or 11 .

If we exclude 11, triangles o hexagons must be in combination with squares, and we have seen that squares can never be introduced if we exclude 4 and either 5 or 6 . But 1 may be continued by 11 (fig. 16).

(g) We must have either 11 , or 10 and 12 .

For, excluding 11, we must have 12 at least, for dodecagons are only given by 7,11 , and 12 , and 7 is excluderl by $\left(f^{\circ}\right)$.

Starting therefore with 12, we must have tig. 13. The concavities can now be filled either with dodecagons, or with squares and triangles. The latter gives 10 , the former never introduces triangles.

(h) If 4 and 12 be excluded, the only combination is $7,10,11$.

Excluding 4 and 12, we must have 11 . Let us start therefore with fig. 14 (the heavy lines). a cannot be a spuare, for that gives a hexagon at $b$; nor a hexagon; nor a dodecagon, since 12 is excluded. Hence $a$ must be a triangle, and we get the figure with dotted lines.

Again, if we start with fig. 15 (the heavy lines), it must be continued as in the dotted lines.

Lastly, let us start with fig. 16. A dodecagon at a gives us a variation of fig. 14 with the hexagon turned through $60^{\circ}$, a square gives fig. 15, and a triangle fig. 16 with the dotted lines.

The continuation of any of these figures (under the given conditions) will introduce no angles other than 1, 7, 10,11; and fig. 16 must be excluded, since it does not contain hexagons. Hence the only possible combination is $7,10,11$.

(i) If 4 and 10 be excluded, the only combination is $1,11,12$.

We must have 12 , by $(h)$; and 11 , by $(g)$.

Starting with 12 we get fig. 17. At $a$ we must put either a hexagon or six triangles, hence the figure can only be continued as in the dotted lines, where some of the hexagons, but not all, must be filled up with triangles. This is the combination $1,11,12$.

Rejecting according to these rules, there are left 222 combinations. I have tested 
these and found composite types corresponding to 176 of them. Of the remainder it is probable that a considerable proportion do not give types. Thus it seems probable that the only types which involve 11 without 4 are $1,10,11,12 ; 1,11,12 ; 7,10,11$; and $7,10,11,12$.

The combinations are all inclurled in the following lists. A. contains the 222 left after rejection, B. those which I have not verified.

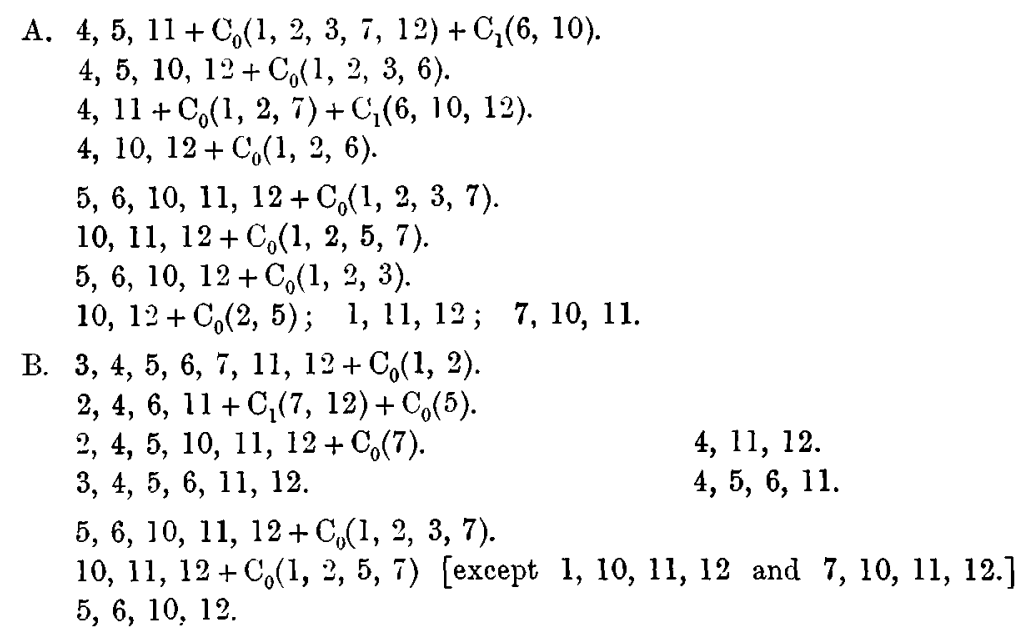

$\S 17$. Many of these comprsite types can be obtained from the simple types by filling up the hexagons and ridecagons. Thus, as we have seen, the type $1,11,12$ can be obtained from the simple type 12 by filling up some of the hexagons with triangles. From the same simple type can be obtained nine other composite types involving the nodes $1,4,10,11,12$. In the same way, having obtained one example of one type, it is generally possible to obtain a number of other types from it by some simple substitutions or displacements. A classification of the composite types might thus be attempted, based upon their structure. In this way types which are widely separated in the present classification would be brought together, and vice versa. It is to be noted, however, that the general variety of a type may fall on the lines of no simple network, so that a classification such as that suggested would be difficult to apply in the general case.

\section{The Elliptic Plane.}

$\S 18$. We proceed to investigate the semi-regular networks upon the elliptic plane, or, what is the same thing, upon the sphere, or in general upon a closed surface of constant positive curvature.

We shall first find what species of nodes are possible. Since the angle of a regular polygon here depends upon the area of the figure, it is obvious that the number of species of nodes is infinite. Whatever holds on the Euclidean plane regarding the number of polygons which can meet at a point will hold a fortiori for the sphere. Hence we have only two cases to consider: viz., at a point there may be $1^{\circ}$ two, $2^{\circ}$ three 
different kinds of polysoms, but not more. The species of nodes thus fall under three classes, Class A. consisting of the hommonerous norles. Wo shall take the other two classes separately, and find those moles which give simple types.

$\S 19$. Class B. Let there be $\eta_{1} \eta_{1}-$ gons and $\eta_{2} n_{2}$-gons at a point; then, if $a_{1}, a_{2}$ are the angles of the $n_{1}$ - and $n_{2}-$ gom,

Also

$$
\begin{aligned}
& n_{1} a_{1}>\left(n_{1}-2\right) \pi . \\
& p_{1} \alpha_{1}+p_{2} \alpha_{2}=2 \pi .
\end{aligned}
$$

Therefore

$$
\begin{aligned}
& p_{1}\left(1-\frac{2}{n_{1}}\right)+p_{2}\left(1-\frac{\ddot{*}}{\eta_{2}}\right)<\because \text {, } \\
& n_{1} n_{2}(2-p)+2\left(p_{1} n_{2}+p_{2} n_{1}\right) \equiv د>0 .
\end{aligned}
$$

or,

Giving $\Delta$ positive integral values we get a series of equations to solve under the following conditions: $n_{1} \neq n_{2}>3,3 \ngtr p \ngtr 5$, therefore $p_{1}, p_{2} \ngtr 4$. Also, if $p_{1}=2, p_{2}=3$, the smallest values of $n_{1}$ and $n_{2}$ which are pissible are $n_{1}=4, n_{2}=3$, but these make $\Delta=0$, hence the only possible sets of values of $p_{1}$ and $p_{2}$ are

$$
\begin{aligned}
& p_{1}=1,1,1,2, \\
& p_{2}=2,3,4, \because .
\end{aligned}
$$

Further, if $p_{1}=1, p_{2}=2$, in order that the node may give a simple type, $n_{2}$ must be even, for the $n_{2}$-gon must be surrounded alternately with $n_{1}$-gons and $n_{2}$-gons.

We have then

$$
n_{1}=\underset{n_{2}(p-2)-2 p_{1}-2}{n_{2}}
$$

We shall take each set of values of $p_{1}, p_{2}$ in turn.

$$
\begin{aligned}
& \left.\begin{array}{l}
p_{1}=1 \\
p_{2}=2
\end{array}\right\} \quad \quad{ }_{1}=\frac{2 n_{2}-\Delta}{n_{2}-4}=2+\frac{8}{n_{2}}-4 . \\
& \Delta=2 . \quad n_{2}=6,10 \quad \Delta=6 . \quad n_{2}=6 \\
& n_{1}=5,3 \quad n_{1}=3 \\
& د=4 . \quad n_{2}=6,8 \quad \Delta=8 . \quad n_{2}=4 \\
& n_{1}=4,3 \quad n_{1}=\text { any integer. } \\
& \left.\begin{array}{l}
\mu_{1}=1 \\
p_{2}=3
\end{array}\right\} \quad n_{1}=\frac{2 n_{2}-\Delta}{2} n_{2}-6=1+\frac{3-\frac{1}{2} \Delta}{n_{3}-3} \\
& \Delta=2 . \quad n_{2}=4 \\
& n_{1}=3 \\
& \Delta=4 \quad \text { impossible } \\
& د=6 . \quad n_{2}=3 \\
& n_{1}=\text { iny integer. } \\
& \left.\begin{array}{l}
p_{1}=1 \\
p_{2}=4
\end{array}\right\} \\
& n_{1}=\frac{2 n_{2}-\Delta}{3 n_{2}-8} \\
& \text { or } 3 n_{1}=2+\frac{16-3 \Delta}{3 n_{2}-8} \text {. } \\
& \Delta=1 . \quad n_{2,2}=3 \\
& n_{1}=5 \\
& \Delta=2 . \quad n_{2}=3 \\
& {\left[\Delta=3 . \quad n_{2}=3=n_{1}, \text { excluded. }\right]} \\
& \Delta \ngtr 3 .
\end{aligned}
$$




$$
\begin{array}{lcc}
\left.\begin{array}{l}
p_{1}=2 \\
p_{2}=2
\end{array}\right\} & & n_{1}=\frac{4 n_{2}-\Delta}{2 n_{2}-4}=2+\frac{4-\frac{1}{2} \Delta}{n_{2}-2} \\
& & \\
& & {\left[\Delta=6 . \quad n_{2}=3=n_{1}, \text { excluded. }\right]} \\
& n_{2}=3 \\
n_{1}=5 & \\
n_{2}=3 & \Delta \ngtr 6 .
\end{array}
$$

$\$ 20$. Class C. We have here the equation

$$
\begin{gathered}
p_{1}\left(1-\frac{2}{n_{1}}\right)+p_{2}\left(1-\frac{2}{n_{2}}\right)+p_{3}\left(1-\frac{2}{n_{3}}\right)<2, \\
\text { or } \quad n_{1} n_{2} n_{3}(2-p)+2\left(p_{1} n_{2} n_{3}+p_{2} n_{3} n_{1}+p_{3} n_{1} n_{2}\right) \equiv \Delta>0 .
\end{gathered}
$$

We have to solve this equation under the following conditions: $n_{1} \neq n_{2} \neq n_{3}>3$, $3 \ngtr p \ngtr 4$, therefore $p_{1}, p_{2}, p_{3} \ngtr 2$, and we cannot have $n_{1}=5, n_{2}=6, n_{3}=7$ together.

Further, if $p_{1}=p_{2}=p_{3}=1, n_{1}, n_{2}$, and $n_{3}$ must all be even, for the $n_{1}$-gon must be surrounded alternately with $n_{2}$-gons and $n_{3}$-gons. Again, if $p=4, n_{3}=3, p_{3}=2$, then starting with an $n_{1}$-gon we must have on successive sides an $n_{2}$-gon, a double triangle, a double triangle, an $n_{2}$-gon, and so on (fig. 18). Hence $n_{1}$, and similarly $n_{2}$, must be a multiple of 3 .

The only possible sets of values of $p_{1}, p_{2}, p_{3}, n_{3}$ are therefore

We have then

$$
\begin{aligned}
& p_{1}=p_{2}=p_{3}=1, n_{3}=4 ; \\
& p_{3}=2, p_{1}=p_{2}=1, n_{3}=3 \text { or } 4 .
\end{aligned}
$$

$$
\begin{aligned}
& n_{1}=\frac{2 n_{2} n_{3} p_{1}-\Delta}{n_{2}\left(\overline{p-2} \cdot n_{3}-2 p_{3}\right)-2 p_{2} n_{3}} \\
& \left.\begin{array}{l}
p_{1}=p_{2}=p_{3}=1 \\
n_{3}=4
\end{array}\right\} \quad n_{1}=\frac{8 n_{2}-\Delta}{2 n_{2}-8}=4+\frac{16-\frac{1}{2} \Delta}{n_{2}-4}
\end{aligned}
$$

Since $n_{2}$ and ${ }_{1}$ are both even, $\Delta$ must be a multiple of 8 .

$$
\begin{aligned}
& \Delta=8 . n_{2}=6 \quad\left[\Delta=24 . n_{2}=6=n_{1}, \text { excluded. }\right] \\
& \Delta=16 . \begin{array}{l}
n_{1}=10 \\
n_{2}=6 \\
n_{1}=8
\end{array} \quad\left[\Delta=32 . n_{2}=4, \text { excluded. }\right] \\
& \left.\begin{array}{l}
p_{1}=p_{2}=1 \\
p_{3}=2 \\
n_{2}=3
\end{array}\right\} \quad n_{1}=\frac{6 n_{2}-\Delta}{2 n_{2}-6}=3+\frac{9-\frac{1}{2} \Delta}{n_{2}-3} .
\end{aligned}
$$

Since $n_{2}$ and $n_{1}$ are both multiples of $3, \Delta$ must be a multiple of 18 , but $\Delta=18$ gives $n_{2}=3$ and any higher multiple is excluded, hence there are no developable nodes of this type.

$$
\begin{aligned}
& \left.\begin{array}{l}
p_{1}=p_{2}=1 \\
p_{3}=2 \\
n_{3}=4
\end{array}\right\} \quad n_{1}=\begin{array}{l}
8 n_{2,}-\Delta \\
4 n_{2}-8
\end{array}=2+\frac{4-\frac{1}{4} \Delta}{n_{2}-2} . \\
& \left.\Delta=4 . \begin{array}{ll}
n_{2}=: 3 \\
n_{1}=5
\end{array} \quad\left[\Delta=8, \quad \begin{array}{l}
n_{2}=3 \\
n_{1}=4
\end{array}\right\} \text { excluded. }\right] \\
& {\left[\Delta=12 . \quad n_{2}=3=n_{1} \text {, excluded. }\right]}
\end{aligned}
$$


$\S 21$. We have found, then, the following developable species of nodes:

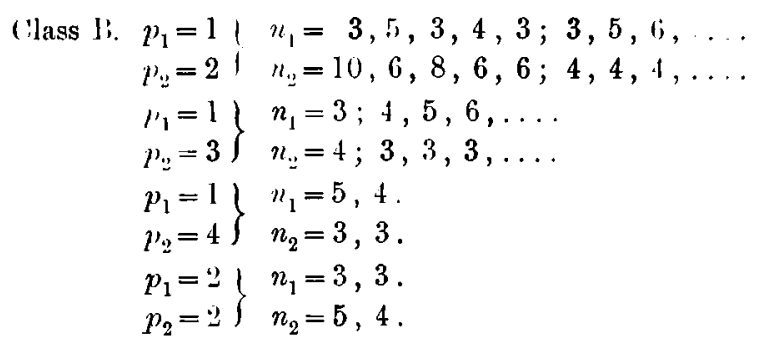

Class C. $p_{1}=1, n_{1}=4,4$.

$p_{2}=1 ; n_{2}=6,6$

$p_{3}=1 \quad n_{3}=10,8$.

$\left.p_{1}=1\right) n_{1}=3$.

$\left.p_{2}=1\right\} n_{\mathrm{2}}=5$.

$\left.\nu_{3}=2\right\} n_{3}=4$.

Each of these gives a simple type of network.

$\S 22$. We shall find the number of polygons, lines, and points in the complete network.

Let $\mathrm{N}_{2}^{\prime}, \mathrm{N}_{2}^{\prime \prime}, \mathrm{N}_{2}^{\prime \prime \prime}$ be the number of $n^{\prime}-, u^{\prime \prime}-$, and $n^{\prime \prime \prime}$-gons, $\mathrm{N}_{2}, \mathrm{~N}_{1}, \mathrm{~N}_{0}$ the number of polygons, lines, and points respectively.

Then $(\$ \S 11,12)$

$$
\begin{aligned}
& \mathrm{N}_{2}=\mathrm{N}_{2}^{\prime}+\mathrm{N}_{2}^{\prime \prime}+\mathrm{N}_{2}^{\prime \prime \prime} \quad \text {. . . . . . . . . . . . (1) } \\
& n^{\prime} \mathrm{N}_{0}^{\prime}=j^{\prime} \mathrm{N}_{0} \\
& \left.\begin{array}{l}
n^{\prime \prime} \mathrm{N}_{2}^{\prime \prime}=p^{\prime \prime} \mathrm{N}_{0} \\
n^{\prime \prime \prime} \mathrm{N}_{2}^{\prime \prime \prime}=p^{\prime \prime \prime} \mathrm{N}_{0}
\end{array}\right\} \\
& p \mathrm{~N}_{0}=2 \mathrm{~N}_{1}
\end{aligned}
$$

where $p=p^{\prime}+p^{\prime \prime}+p^{\prime \prime \prime}$.

Finally, EvLer's polyhedral formula is

$$
\mathrm{N}_{2}-\mathrm{N}_{1}+\mathrm{N}_{0}=2
$$

From (2) and (1) we get

and from (3)

$$
\mathrm{N}_{2}=\left(\frac{p^{\prime}}{n^{\prime}}+\frac{p^{\prime \prime}}{n^{\prime \prime}}+\frac{p^{\prime \prime \prime}}{n^{\prime \prime \prime}}\right) \mathrm{N}_{0}
$$

Substituting in (4),

$$
\mathrm{N}_{1}=\frac{p}{2} \mathrm{~N}_{0} .
$$

whence

$$
\left(\frac{p^{\prime}}{n^{\prime}}+\frac{p^{\prime \prime}}{n^{\prime \prime}}+\frac{p^{\prime \prime \prime}}{n^{\prime \prime \prime}}-\frac{p}{2}+1\right) \mathrm{N}_{0}=2
$$

Hence

$$
\mathrm{N}_{11}=\frac{4 n^{\prime} n^{\prime \prime} n^{\prime \prime \prime}}{\Delta} \text {. }
$$

$$
\begin{gathered}
\mathrm{N}_{1}=\frac{2 n^{\prime} n^{\prime \prime} n^{\prime \prime} p}{\Delta} . \\
\mathrm{N}_{2}^{\prime}=\frac{4 p^{\prime} n^{\prime \prime} n^{\prime \prime \prime}}{\Delta}, \mathrm{N}_{2}^{\prime \prime}=\frac{4 p^{\prime \prime} n^{\prime \prime \prime} n^{\prime}}{\Delta}, \mathrm{N}_{2}^{\prime \prime \prime}=\frac{4 \mu^{\prime \prime \prime} n^{\prime} n^{\prime \prime}}{\Delta} .
\end{gathered}
$$


For Class B. the corresponding formulæ are

$$
\begin{aligned}
& \mathrm{N}_{0}=\frac{4 n^{\prime} n^{\prime \prime}}{د}, \mathrm{~N}_{1}=\frac{2 n^{\prime} n^{\prime \prime} p}{\Delta} ; \\
& \mathrm{N}_{2}^{\prime}=\frac{4 p^{\prime} n^{\prime \prime}}{\Delta}, \mathrm{N}_{2}^{\prime \prime}=\frac{4 p^{\prime \prime} n^{\prime}}{د},
\end{aligned}
$$

where $\Delta=n^{\prime} n^{\prime \prime}(2-p)+2\left(p^{\prime} n^{\prime \prime}+p^{\prime \prime} n^{\prime}\right)$.

\section{The Simple Types.}

$\S 23$. We proceed now to classify the simple types and investigate their varieties. The division into classes according to the number of kinds of polygons can still be made, but the subdivision into groups according to the kinds of polygons involved is useless, as there are an infinite number of kinds of polygons. We shall therefore, for the present, classify them according to the types of nodes. I consider two nodes to be of the same type when the values of $p_{1}, p_{2}, p_{3}$ are the same for both. A network will not in general admit of variation unless its node does so. But this rule is not always true; e.g. the type of angle $\mathrm{PQ}_{3}$ is unvaried, but, as we shall see, one of the networks corresponding to this type admits of two distinct varieties. We shall give for each network the values of $\mathrm{N}_{0}, \mathrm{~N}_{1}, \mathrm{~N}_{2}$, etc. Unless otherwise specified, the network is unique.

Class B. I. $p^{\prime}=1, p^{\prime \prime}=2$.

$$
\begin{aligned}
& \text { (1) } \Delta=2 \text {. (a) } n^{\prime}=3, \mathrm{~N}_{2}^{\prime}=20 \\
& n^{\prime \prime}=10, \mathrm{~N}_{2}^{\prime \prime}=12 \\
& N_{0}=60, N_{1}=90, N_{2}=32 \text { (fig. 19). } \\
& \text { (b) } n^{\prime}=5, N_{2}^{\prime}=12 \\
& n^{\prime \prime}=6, \mathrm{~N}_{2}^{\prime \prime}=20 \\
& \mathrm{~N}_{0}=60, \mathrm{~N}_{1}=90, \mathrm{~N}_{2}=32 \quad \text { (fig. 21). }
\end{aligned}
$$

(2) $\Delta=4$

$$
\begin{aligned}
& \text { (a) } n^{\prime}=3, \mathrm{~N}_{2}{ }^{\prime}=8 \\
& n^{\prime \prime}=8, \mathrm{~N}_{2}^{\prime \prime}=6 \\
& \mathrm{~N}_{0}=24, \mathrm{~N}_{1}=36, \mathrm{~N}_{2}=14 \quad \text { (fig. 20). }
\end{aligned}
$$

(b) $n^{\prime}=4, \mathrm{~N}_{2}^{\prime}=6$

$n^{\prime \prime}=6, \mathrm{~N}_{2}^{\prime \prime}=8$

$$
\mathrm{N}_{0}=24, \mathrm{~N}_{1}=36, \mathrm{~N}_{2}=14 \quad \text { (fig. 22). }
$$

(3) $\Delta=6 . \quad n^{\prime}=3, \mathrm{~N}_{2}^{\prime}=4$

$$
\begin{aligned}
& n^{\prime \prime}=6, \mathrm{~N}_{2}^{\prime \prime}=4 \\
& \mathrm{~N}_{0}=12, \mathrm{~N}_{1}=18, \mathrm{~N}_{2}=8 \quad \text { (fig. 23). }
\end{aligned}
$$

(4) $\Delta=8$.

$$
\begin{aligned}
& n^{\prime \prime}=4, \mathrm{~N}_{2}^{\prime \prime}=n . \\
& n^{\prime}=n, \mathrm{~N}_{2}^{\prime}=2 . \\
& \mathrm{N}_{0}=2 n, \mathrm{~N}_{1}=3 n, \mathrm{~N}_{2}=n+2 \quad \text { (fig. } 24 \text { ). }
\end{aligned}
$$

II. $p^{\prime}=1, p^{\prime \prime}=3$.

$$
\text { (1) } \begin{aligned}
& \Delta=2 . n^{\prime}=3, \mathrm{~N}_{2}^{\prime}=8 . \\
& n^{\prime \prime}=4, \mathrm{~N}_{2}^{\prime \prime}=18 . \\
& \mathrm{N}_{0}=24, \mathrm{~N}_{1}=48, \mathrm{~N}_{2}=26 .
\end{aligned}
$$

This type has two varieties. It contains a group formed by a quadrilateral surrounded 
alternately with quadrilaterals and trimits. The boundary of this group is a renllin octagon, and by turning it round throushl $\frac{\pi}{4}$ we get the other viricty (figs. 26, 27).

(2) $\Delta=6 . \quad n^{\prime \prime}=3, \mathrm{~N}_{2}^{\prime \prime}=2 n$

$$
\begin{aligned}
& n^{\prime}=n, \mathrm{~N}_{2}^{\prime}=2 \\
& \quad \mathrm{~N}_{11}=2 n, \mathrm{~N}_{1}=4 n, \mathrm{~N}_{2}=2(n+1) \quad \text { (fig. 25). }
\end{aligned}
$$

III. $p^{\prime}=2, p^{\prime \prime}=2$.

$$
\begin{array}{ll}
\Delta=2 . & n^{\prime}=3, \mathrm{~N}_{2}^{\prime}=20 \\
& n^{\prime \prime}=5, \mathrm{~N}_{2}^{\prime \prime}=12 \\
& \mathrm{~N}_{0}^{\prime}=30, \mathrm{~N}_{1}=60, \mathrm{~N}_{2}=32 .
\end{array}
$$

A certain great circle divides this network into two equal groups. By turning one of these through $\frac{\pi}{5}$ a second variety is obtained (figs. 28, 29).

$$
\text { (2) } \begin{array}{ll}
\Delta=4 . & n^{\prime}=3, \mathrm{~N}_{2}^{\prime}=8 \\
n^{\prime \prime}=4, N_{2}^{\prime \prime}=6 \\
\mathrm{~N}_{0}=12, \mathrm{~N}_{1}=24, \mathrm{~N}_{2}=14 .
\end{array}
$$

Like the preceding, this network has two varieties which may be obtained in a similar way, viz., by turning one of the groups through $\frac{\pi}{3}$ (figs. 30,31 ).

IV. $p^{\prime}=1, p^{\prime \prime}=4$.

$$
\begin{array}{ll}
\text { (1) } \Delta=1 . & n^{\prime}=5, \mathrm{~N}_{2}^{\prime}=12 \\
& n^{\prime \prime}=3, \mathrm{~N}_{2}^{\prime \prime}=80 \\
& \mathrm{~N}_{0}=60, \mathrm{~N}_{1}=150, \mathrm{~N}_{2}=92 \quad \text { (fig. 32). } \\
\text { (2) } \Delta=2 . & n^{\prime}=4, \mathrm{~N}_{2}^{\prime}=6 \\
& n^{\prime \prime}=3, \mathrm{~N}_{2}^{\prime \prime}=32 \\
& \mathrm{~N}_{0}=24, \mathrm{~N}_{1}=60, \mathrm{~N}_{2}=38 \quad \text { (fig. 33). }
\end{array}
$$

These two networks are asymmetrical. Each exists in two forms which are enantiomorph. 'The one could be obtained from the other by turning the sphere inside out, supposing this to be possible, as it would be in space of four dimensions.

§24. Class C. I. $p^{\prime}=1, p^{\prime \prime}=1, p^{\prime \prime \prime}=1$.

$$
\begin{aligned}
& \text { (1) } \Delta=8, \quad n^{\prime}=4, \quad \mathrm{~N}_{2}^{\prime}=30 \\
& n^{\prime \prime}=6, \quad \mathrm{~N}_{2}^{\prime \prime}=20 \\
& n^{\prime \prime \prime}=10, \mathrm{~N}_{2}^{\prime \prime \prime}=12 \\
& \text { (2) } \Delta=16, n^{\prime}=4, \mathrm{~N}_{2}^{\prime}=12 \\
& \mathrm{~N}_{0}=120, \mathrm{~N}_{1}=180, \mathrm{~N}_{2}=62 \quad \text { (fig. 34). } \\
& n^{\prime \prime}=6, \mathrm{~N}_{2}^{\prime \prime}=8 \\
& u^{\prime \prime \prime}=8, \mathrm{~N}_{2}^{\prime \prime \prime}=6 \\
& \mathrm{~N}_{0}=48, \mathrm{~N}_{1}=72, \mathrm{~N}_{2}=26 \quad \text { (fig. 35). }
\end{aligned}
$$

II. $p^{\prime}=1, p^{\prime \prime}=1, p^{\prime \prime \prime}=2$.

$$
\begin{aligned}
& \Delta=4, n^{\prime}=3, \mathrm{~N}_{2}^{\prime}=20 \\
& n^{\prime \prime}=5, \mathrm{~N}_{2}^{\prime \prime}=12 \\
& n^{\prime \prime \prime}=4, \mathrm{~N}_{2}^{\prime \prime \prime}=30 \\
& \quad \mathrm{~N}_{0}=60, \mathrm{~N}_{1}=120, \mathrm{~N}_{2}=62 .
\end{aligned}
$$

Of this type there are five varieties, which may be obtained as follows:- In one if the varieties (fig. 36) there are no two quadrilaterals adjacent. Each pentagon has a quadrilateral on each of its sides and forms the centre of a group with a regular decagon as boundary. Let us call TRANS. ROY. SOC. EDIN., VOL. KLI. PART III. (NO. 29). 
this the fundamental variety. Then all the other varieties can be oltained from it by turning some of the groups through $\frac{\pi}{5}$. Let us denote this operation by $R$. In the fundamental variety the twelve pentagons occupy relatively the same positions as the meshes of the dodecahedral network, so that with respect to one of the groups the others can be divided into three sets: 5 adjacent, 5 circumjacent, and 1 opposite. Now suppose the operation $\mathrm{R}$ to be performed upon one of the groups. This gives a variety $\beta_{1}$ (fig. 37 ). Next suppose a second group to he operated upon. The adjacent ones cannot be moved, for the first operation has destroyed their symmetry. Operating upon the opposite one we get a variety $\beta_{2}$ (fig. 38), while operating upon one of the circumjacent groups we get a fourth variety $\gamma_{1}$ (fig. 39). From $\beta_{2}$ we cannot obtain any further variety, for each of the remaining groups is adjacent to one of those already operated on. From $\gamma_{1}$ we can obtain a fifth variety, $\gamma_{2}$ (fig. 40 ), by turning either of the two groups which are circumjacent to both. In $\beta_{1}$ and $\beta_{2}$ pairs of quadrilaterals occur, 5 in the former, 10 in the latter. In $\gamma_{1}$ and $\gamma_{2}$ there occur respectively 1 and 3 groups of three quadrilaterals.

$\$ 25$. To every spherical network there corresponds a convex polyhedron whose vertices are the nodes of the network. The polyhedra which correspond to the semiregular networks have for their faces regular plane polygons. These form only a class of convex polyherlra in general, but they are the only ones whose faces may be regular polygons, and which, at the same time, may be inscribed in a sphere.

If we examine the numbers of the several polygons in the various networks above we find that, with the exception of the two infinite series, they can all be connected with the regular networks. 'The series with two quadrilaterals and an $n$-gon at each point corresponds to a series of right prisms on a regular polygonal base, the altitude diminishing indefinitely as $n$ increases.

The polyhedra corresponding to the other types can be obtained from the regular polyhedra by cutting off the corners in particular ways. Thus the octahedron (fig. 23) bounded by triangles and hexagons can be obtained from the regular tetrahedron by cutting off the corners, either triangles or hexagons corresponding to vertices, according to the depth of the section. When the numbers of the polygons are the numbers of faces, lines or vertices of a regular polyhedron, it is evident in what way they correspond. In some, however, the same kind of polygon may correspond to both edges and vertices, then its number has to be livided into two parts, each a multiple of the number of edges or vertices of the regular polyhedron.

This holds only for the unique types and the fiudamental varieties of the other types, i.e. those in which no two polygons of the same kind are adjacent. The other varieties may or may not be obtainable from the corresponding regular polyhedron. Those of Class B. are not, while the four derived varieties of Class C. II. may still he obtained from the regular dodecahedron, since the positions of the pentagons are unchanged.

$\$ 26$. We may therefore group the simple types in three divisions according to their morphology. We shall use the notation $3_{1}{ }^{1} \mathbf{6}_{2}{ }^{k}$ to renote a simple type consisting

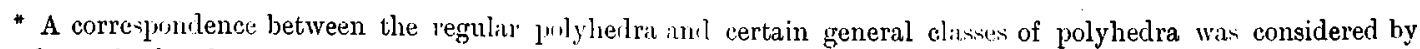

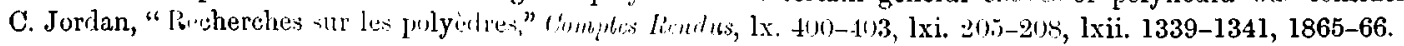


of triangles and hexalnons, where the subscript refers to the number of polygons at a point, and the index to the number of polygons in the whole network.

I. Tetrahedral. $\quad 3_{1}{ }^{4} 6_{2}{ }^{4}$.

II. Hexahedral. $\quad 3_{1}{ }^{\mathrm{s}} \mathbf{8}^{\circ}{ }^{\circ}$

$$
\begin{aligned}
& 4_{1}{ }^{6} 6_{2}{ }^{\circ}, \quad \quad 3{ }^{8} 4_{2}{ }^{10} \\
& 4_{1}{ }^{11} 6_{1}{ }^{\mathrm{s}} 8_{1}{ }^{6} \text {. }
\end{aligned}
$$

III. Dodecahedial. $\quad 3_{1}{ }^{20} 10_{2}{ }^{12}$,

$$
\begin{aligned}
& 5_{1}{ }^{12} 6_{2}{ }^{201}, \quad 3^{20} 5_{2}{ }^{12} \text {. } \\
& 4_{1}{ }^{30} 6_{1}{ }^{20} 10_{1}{ }^{12}, \quad 3_{1}{ }^{20} 5_{1}{ }^{1 *} 4_{4}{ }^{301} \text {. }
\end{aligned}
$$

With two exceptions, to each hexahedral network there corresponds a dodecahedral one, each pair being obtained in a similar way from the regular network. Thus $3_{1}{ }^{5} 8_{2}{ }^{6}$ and $3_{1}{ }^{20} 10_{2}{ }^{12}$ are obtained by shallow sections from the cube and the dodecahedron respectively; $3_{2}{ }^{8} 4_{2}{ }^{6}$ and $3_{2}{ }^{20} 5_{2}{ }^{12}$ by sections through the middle points of the sides, and so on. There is no dodecahedral network corresponding to $3_{1}{ }^{8} 4_{3}{ }^{18}$. nor is there a hexahedral network corresponding to $3_{1}{ }^{20} 5_{1}{ }^{12} 4_{2}{ }^{30}$. It may be noticed that the values of $\Delta$ for corresponding networks bear the same ratio as the values of $\Delta$ for the regular networks, viz., $1: 2$.

In representing the networks upon the Euclidean plane the method of stereographic projection has been employed, though in some cases, in order to avoid undue crowding towards the centre of the figure, strict stereographic projection has been departed from. For simplicity the nodes are joined by straight lines instead of ares of circles, so that the figures really represent the conical projections of the semi-regular polyhedra.

\section{Composite Types.}

$\S 27$. At first sight it might appear that a very large number of composite types could exist, for there are an infinite number of species of nodes, while on the Euclidean plane where there are a considerable number of composite types there are only a very few species of nodes. A little consideration will show, however. that it is probable that the number of composite types is extremely limited.

Let us take any species of node, $p_{1}, p_{3}, p_{3} ; n_{1}, n_{2}, n_{3}$, and let $\alpha_{1}, a_{2}, a_{3}$ be the angles of the different polygons, $a$ the length of side, $k$ the radius of the sphere.

Then

and

$$
\cos \frac{a}{2 h}=\frac{\cos \frac{\pi}{n_{1}}}{\sin \frac{a_{1}}{2}}=\frac{\cos \frac{\pi}{n_{3}}}{\sin \frac{a_{0}}{2}}=\frac{\cos \frac{\pi}{n_{3}}}{\sin \frac{a}{2}},
$$

$$
p_{1} a_{1}+p_{3} a_{2}+p_{3} a_{3}=2 \pi
$$

These four equations determine $\alpha_{1}, a_{2}, a_{3}$ and $a$. 
Now if an $n_{1}$-gon occurs in the same network in a different combination $p_{1}^{\prime}, p_{2}{ }^{\prime}, p_{4}$; $n_{1}, n_{2}, n_{4}$, then $a_{4}$, the angle of the $n_{4}$-gon, must satisfy the two equations

$$
\cos \frac{a}{2 k}=\frac{\cos \frac{\pi}{n_{n}}}{\sin \frac{a_{4}}{2}}, \quad p_{1}^{\prime} a_{1}+p_{2}^{\prime} \alpha_{2}+p_{4} a_{4}=2 \pi .
$$

If we substitute in the first equation a value of $a_{4}$ corresponding to a possible set of values of $p_{1}{ }^{\prime}, p_{2}{ }^{\prime}, p_{4}$ obtained from the second, we must get an integral value for $n_{4}$. This will not in general happen.

The following negative results may also be obtained at once :

1. No composite types exist with only one kind of polygon, for the angles are all equal, and there must therefore be the same number of polygons at each point.

2. No composite types exist with $p=3$ at each point, for the angle and the side determine $n$.

$\S \approx 8$. A certain number of composite types may be obtained by the following

Theoreu. - Whenever a simple type contains a group of polygons bounded by a regular polygon, the replacement of that group by a single polygon will in general give a composite type.

When such a group occurs it may be replaced by a single polygon, for in the corresponding polyherlron the boundary of the group lies in one plane. Further, the replacement of the group removes at least one line from the nodes at the boundary, and the resulting network contains some nodes with $p$ lines and some with at most $p-1$, i.e. it contains at least two different species of nodes, and is therefore composite.

It follows that, in order that the simple type may give a composite type in this way, $p$ must be $>3$. It may happen that the angle of the boundary polygon is $>180^{\circ}$. If we exclude this case we get the following composite types:

1. From $3_{5}{ }^{20}$ by replacing five covertical triangles by a pentagon.

This may be done in three ways, replacing 1,2 or 3 sets by pentagons.*

The three networks are of different types. They may be denoted as follows, the symbol within brackets denoting the species of node and the coefficient the number of times it occurs in the network.

$$
\begin{array}{lll}
\text { (a) } 6\left(3_{5}\right)+5\left(3_{3} 5_{1}\right) & (\text { fig. } 41) \\
\text { (b) } & 2\left(\mathbf{3}_{5}\right)+6\left(3_{3} 5_{1}\right)+2\left(3_{1} 5_{5}\right) & \text { (fis. } 42) \\
\text { (c) } & 3\left(3_{3} 5_{1}\right)+6\left(3_{1} 5\right) & \text { (fic. } 43)
\end{array}
$$

2. From $3_{1}{ }^{8} 4_{3}{ }^{18}$ by replacing one of the octagonal groups (fig. 27 deleting the part within the heavy lines). $\dagger$.

$$
12\left(3_{1} 4_{3}\right)+8\left(4_{2} 8_{1}\right)
$$

3. From $3{ }_{1}{ }^{20} 5_{1}{ }^{12} 4_{2}{ }^{30}$ (fig. 36) by replacing the decagonal group.

* If two opposite groups of five triangles are replaced by pentagons we get the simple type $\mathbf{3}_{\mathbf{3}} \mathbf{1 0}_{\mathbf{1}}{ }^{\mathbf{2}}$ (like fig, 25).

t If both groups are replaced we get the simple type $44_{2}^{8} 8_{1}^{2}$ (like fig. 24). 
There are eight possible varieties. Let $\mathrm{X}$ denote the original group, $\mathrm{R}$ a group turned through $\frac{\pi}{5}, 1$ the decagon replacing a group, then, if $\mathrm{XY}$ denotes two opposite groups, $\mathrm{Y}$ three groups mutually circumingcent, the valieties can be expressed as follows:

$$
\begin{aligned}
& \text { (a) } 45\left(3_{1} 5_{1} 4_{2}\right)+10\left(4,5_{1} 10_{1}\right) \\
& \begin{array}{ll}
(a) & 0
\end{array}
\end{aligned}
$$

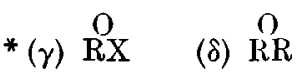

(b) $30\left(3_{1} 5_{1} 4_{1}\right)+20\left(4_{1} 5_{1} 10_{1}\right)$

$$
\text { (a) } \text { OO } \quad(\beta) \stackrel{(}{\mathrm{O}} \quad(\gamma) \stackrel{\mathrm{O}}{\mathrm{OR}}
$$

(c) $15\left(35_{1} 4_{2}\right)+30\left(4_{1} 5_{1} 10_{1}\right)$

OO

$\$ 29$. Further, if we allow angles $>180^{\circ}$ we get the following:

1. From $3_{4}{ }^{8}$, replacing four covertical triangles by a quadrilateral (fig. 45).

$$
\left(3_{4}\right)++\left(3_{2} 4_{1}\right) \text {. Angle of quadrilateral } 180^{\circ} \text {. }
$$

2. From $3_{5}^{20}$ (fig. 44 ).

$\left(3_{5}\right)+5\left(3_{2} 5_{1}\right)$. Angle of pentagon $216^{\circ}$.

3. From $3{ }_{1}{ }^{8} 4_{3}{ }^{18}$ (fig. 27 bounded by the heavy lines).

$$
4\left(3_{1} 4_{3}\right)+8\left(3_{1} 4_{1} \mathbf{8}_{1}\right) \text {. Angle of octagon } 196^{\circ} 50^{\prime} \text {. }
$$

4. From $3_{2}{ }^{8} 4_{2}{ }^{6}$, replacing the hexagonal group (fig. 31 bounded by the heavy lines).

$3\left(3_{2} 4_{2}\right)+6\left(3_{1} 4_{1} 6_{1}\right)$. Angle of hexagon $180^{\circ}$.

5. From $3_{2}{ }^{20} 5_{2}{ }^{12}$, replacing the decagonal group (fig. 29 bounded by the heavy lines).

$10\left(3_{3} 5_{2}\right)+10\left(3_{1} 5_{1} 10_{1}\right)$. Angle of decagon $180^{\circ}$.

6. From $3_{1}{ }^{30} 5_{1}{ }^{12} 4_{2}{ }^{30}$ (fig. 37 deleting the part within the heavy lines).

$5\left(3_{1} 5_{1} 4_{1}\right)+10\left(3_{1} 4_{1} 10_{1}\right)$. Angle of decagon $204^{\circ} 6^{\prime}$.

These are all the composite types obtainable from the simple types. It seems probable that there are no others.

\section{The Hyperbolic Plane.}

$\S 30$. This case does not admit of exhaustive treatment. The number of types of networks is evidently infinite, for there is no limit to the number of lines at a point.

As a rule, the hyperbolic plane contains the types which cannot exist on the Euclidean or the elliptic plane. For example: one $n$-gon and two $2 m$-gons at a point

\footnotetext{
* Asymmetrical. Two enantiomorphic forms, $\stackrel{9}{R X}$ and $\frac{0}{\mathrm{X} R}$.
} 
determine a simple hyperbolic network for all values of $n$ and $m$ for which the network is neither Euclidean nor elliptic. The networks are all infinite.

As regards composite types, we can apply the same remarks as were made in connection with the elliptic networks. The angle of a polygon is determined by the particular combination in which it occurs, and the multiplicity of composite types is thus limited. But, at the same time, it is infinite. For, consider the regular network $\mathbf{3}_{p}(p>6)$ (fig. 46). Any group of $p$ covertical triangles can be replaced by a $p$-gon, so that from this network alone we obtain an infinite number of composite types.

Note added on July 29, 1905.- Since writing the above, I have come across some of the previous work on the subject. The semi-regular polyhedra have long been known. It appears, from the works of PAPPUS of Alexandria and KEPPLER, that they were described in a lost work of ARchimedes.* Pappus $\dagger$ enumerates the series of thirteen (i.e. excluding the two infinite groups, figs. 24 and 25), with the numbers of their faces, edges, and vertices, for which he gives the general formulæ of $\$ 22$. KEPPLER $\ddagger$ establishes them by taking the different possible combinations, first binary and then ternary, containing triangles, squares, and pentagons successively. More recently, accounts of them have been given by MEIER Hirsch $\S$ and R. Baltzer.\| An elaborate article, containing numerous calculations relating to the radius of the circumscribed sphere, inclinations of the faces, etc., was presented by M. VALAT to the French Institute in 1854.T He refers to other writings, in particular to one by Lidonne (1808), but gives no details of them. He shows also how the semi-regular polyhedra are obtained by truncating the Platonic solids. The connection between these polyhedra was also expressed by $\mathrm{K}_{\text {EPPLER }}$ in an ingenious nomenclature which he employed to describe them. The following list of names corresponds to the table on p. 743 ; the numbers refer to the diagrams :-

I. Tetrahedron truncum (23).

II. Cubus truncus (20).

Octahedron truncum (22).

Cuboctahedron truncum (35).

III. Dodecahedron truncum (19).

Icosihedron truncum (21).

Rhombicuboctahedron (26).

Cubus simus (33).

Icosidodecahedron truncum (34).

Cuboctahedron (30).

Icosidodecahedron (28).

Rhombicosidodecahedron (36)

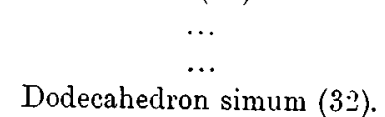

$\cdots$

$\ldots$

In none of these writings is any notice taken of possible varieties, the reason being probably that these varieties do not exhibit the same symmetry as the fundamental varieties. KePpler gives this as the reason for excluding the two infinite series.

* See also T. L. Heath, The Works of Archimedes (Camb. 1897), p. xxxvi.

+ Collectio, lib. v. pars 2.

$\ddagger$ Harmonices Mundr (1619), lib. ii. pp. 61-65.

$\S$ Sammlung geometrischer Aufgctben (Berlin, 1805-7), vol. ii. pp. 139-185.

II Elemente der Mathematik (1862), Bd. ii., Buch v. $\$ 7$.

T Published 1867, under the title "Des Polyèdres semi-réguliers, dits solides d'Archimède," Mém. de lu Soc. des Sciences phys. et nat. de Bordeaux, v. 319-369. 
KePpler has also gone into some detail regarding the Euclidean networks. $\mathrm{He}$ gives* all the developable species of norles and some of the others, with examples of networks formed with them, and other patterns, containing star-polygons, which may be derived from them.

It remains to notice a class of polyhedra connected with the semi-regular polyhedra. ${ }^{\dagger}$ They are obtained by lrawing tangent planes to the circum-sphere at the vertices. To a regular $n$-gon there corresponds then a regular $n$-hedral angle. A regular polyhedion treated in this way gives the conjugate regular polyhedron, but in a semi-regular polyhedron none of the polyhedral angles are regular, and so none of the faces of the "conjugate" polyhedron will be regular polygons. The regular polyhedra have both a circum- and an in-suibed sphere; the semi-regular polyhedra have only a circumscribed sphere, while the conjugate ones have only an inscribed sphere. The corresponding networks are constructed simply by taking as new nodes the centres of the old meshes. The polyhedra conjugate to the findumental varietics have their faces all congruent. This does not holl for the other varieties (with the exception of that corresponding to $3_{1}{ }^{8} 4_{3}{ }^{18}$, fig. 27 ). Two of this class are interesting as being the only ones which have all their edges equal, viz., the rhombohedra formed from the fundamental varieties of $3_{2}{ }^{8} 4_{2}{ }^{6}$ and $3_{2}{ }^{20} 5_{2}{ }^{12}$ (figs. 30 and 28). There is an analogous Euclidean network conjugate to $\mathrm{T}_{2} \mathrm{H}_{2}$, i.e. $\mathbf{3}_{2} \mathbf{6}_{2}$.

$U_{11}$ p. 743 there occurs a misstatement. The hexahedial network $3{ }_{1}{ }^{8} 4_{3}{ }^{6+12}$ (Rhombicuboctahedron), though it contains only two kinds of polygons, really corresponds to the dodecahedral network $3_{1}{ }^{20} 5_{1}{ }^{12} 4_{2}{ }^{30}$ (Rhombicosidodecahedron), being obtained in a similar way from the corresponeling regular network. Thus the correspondence between the hexahedral and the dodecahedlal networks is complete.

* Loc. cit., pp. 51-5.5. p. $3+\%$.

† KepPler and Baltzer, loc. cit.; Meiek Hirsce, loc. cit., pp. 186-195; J. H. L. MüLler, Trigonometrie (185:2), 
Sommerville-Netwokks of the Plane in Absolute Geometry. - Plate I.

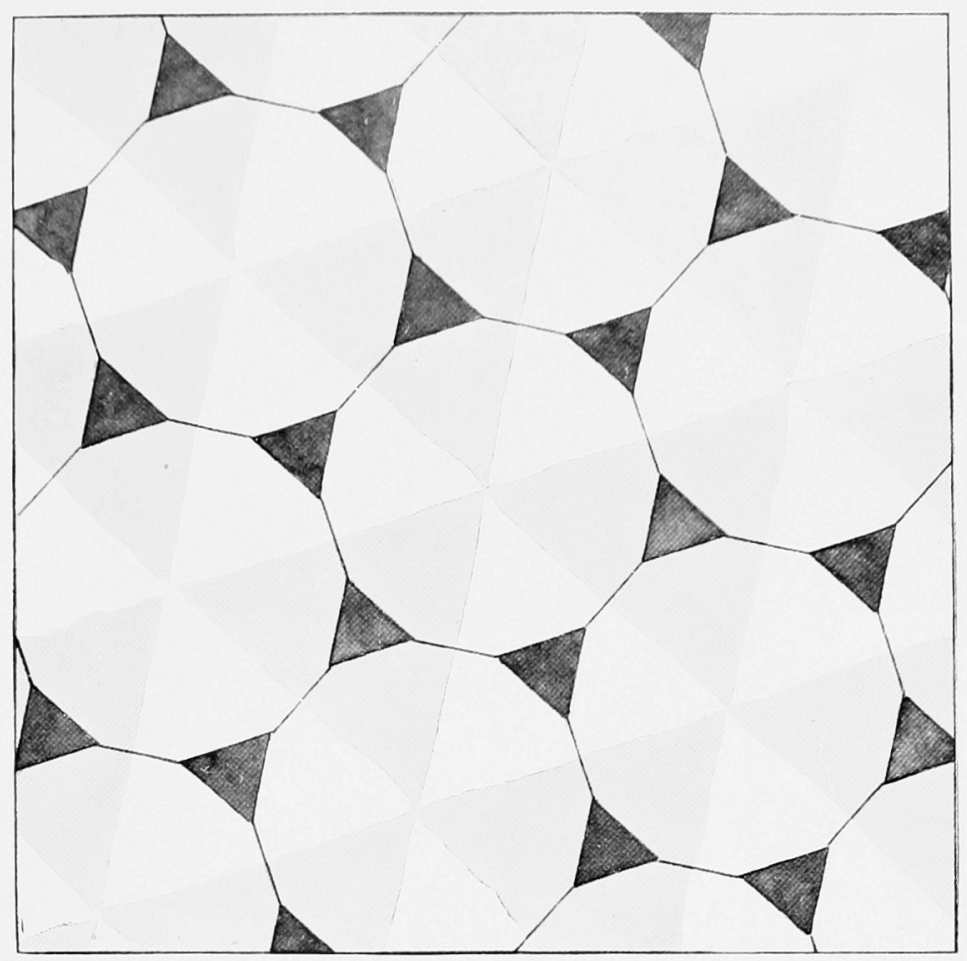

Fig. 2.

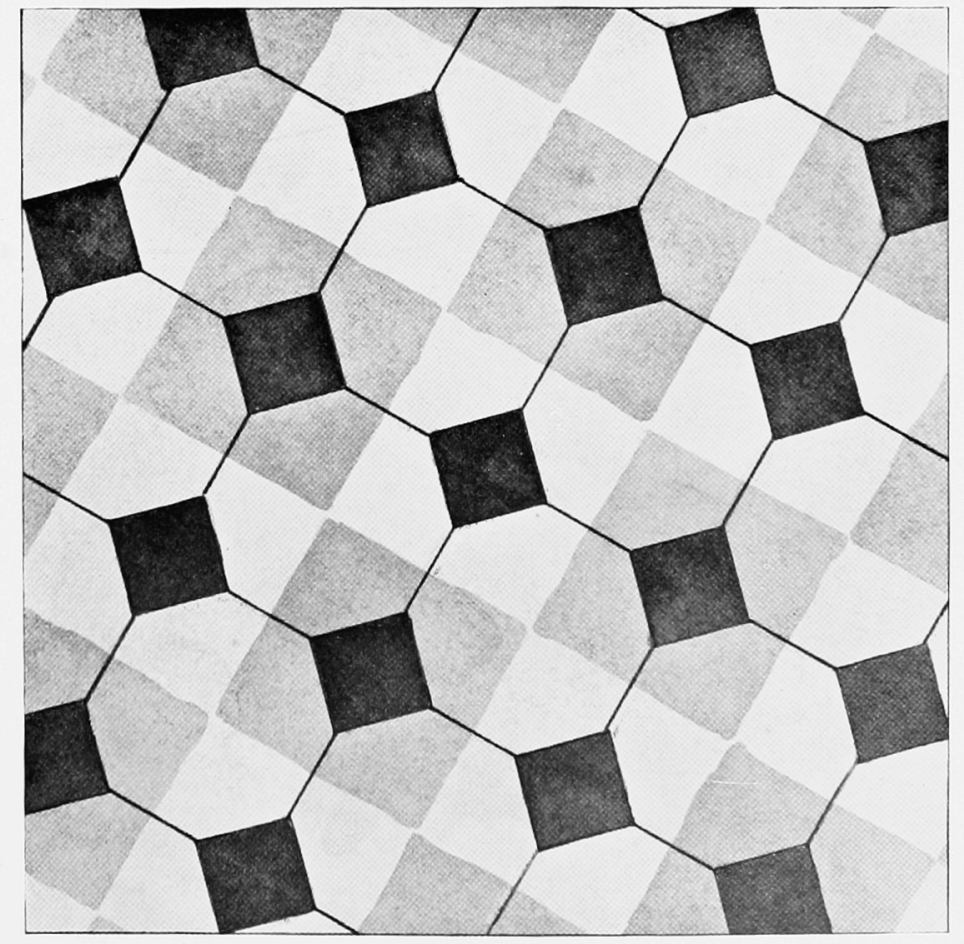

Fig. 3. 
Sommerville - Networks of the Plane in Absolute Geometry.-Plate II.

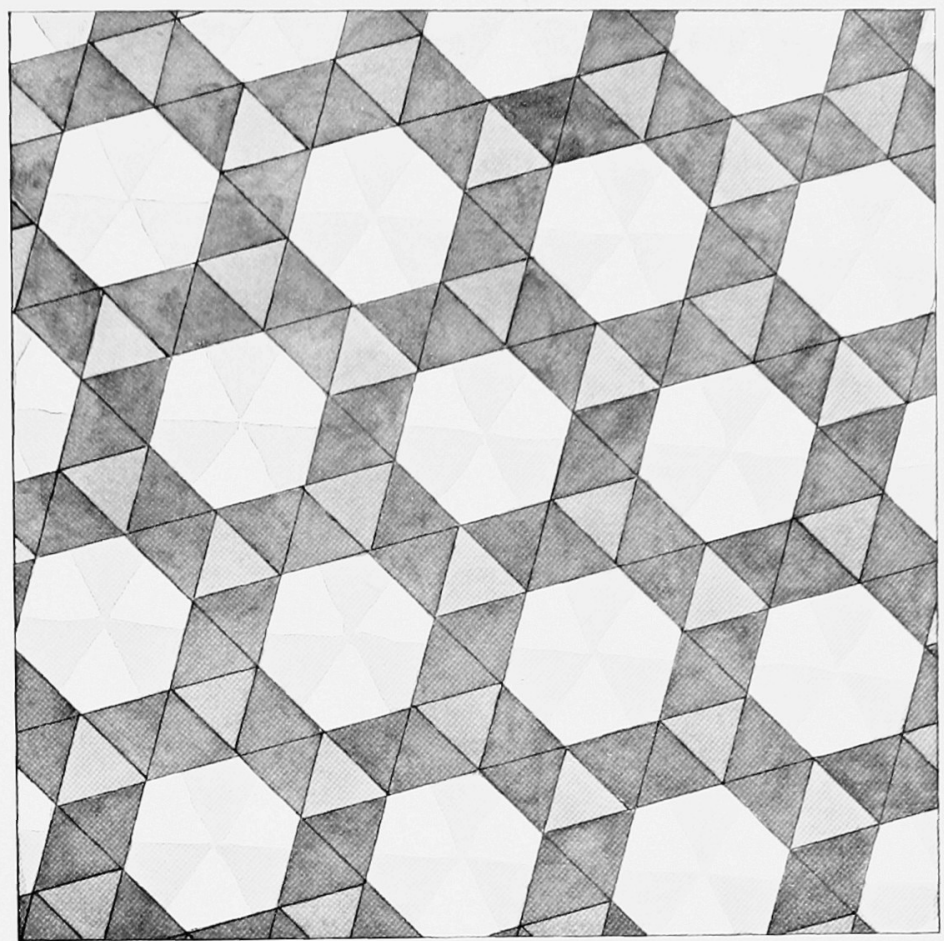

Fig 4 .

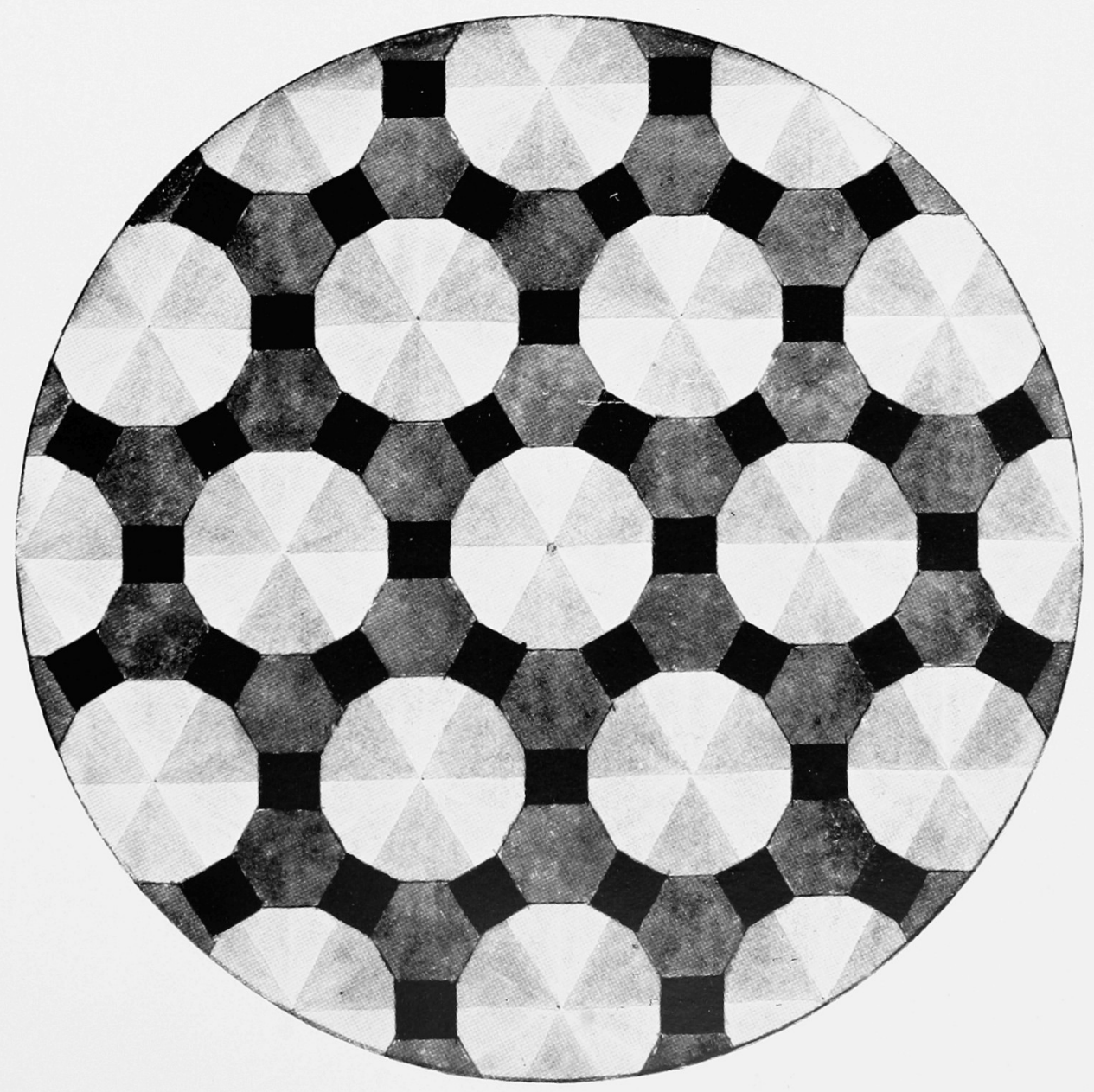

Fig. 5 . 
Trans. Roy. Soc. Edin r.

Vol. XLI.

Sommerville-Networks ol: the Plane in Absolute Geometry.-Plate III.

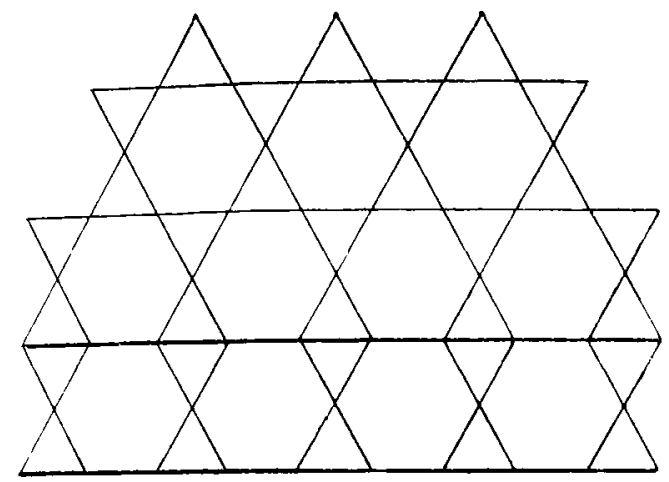

Fig 6.

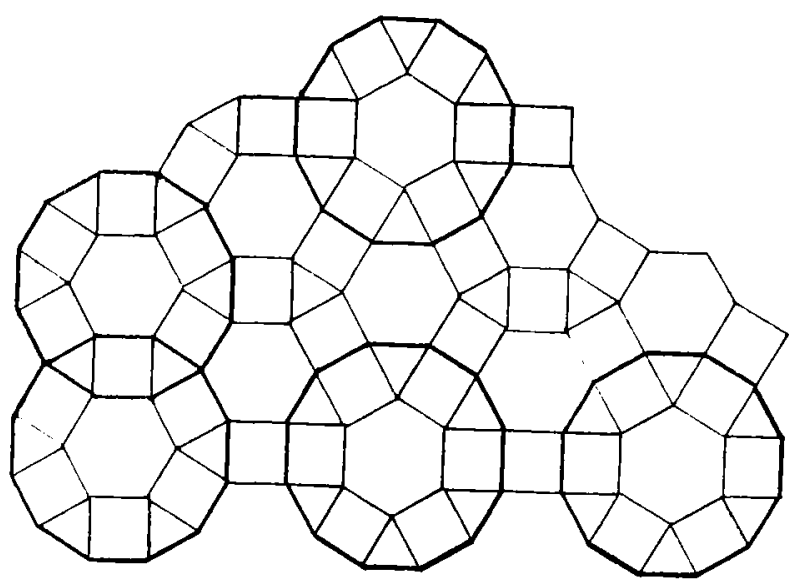

Fig. 7.

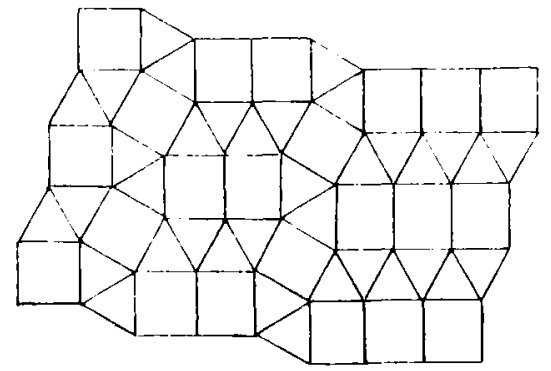

Fig. 8.
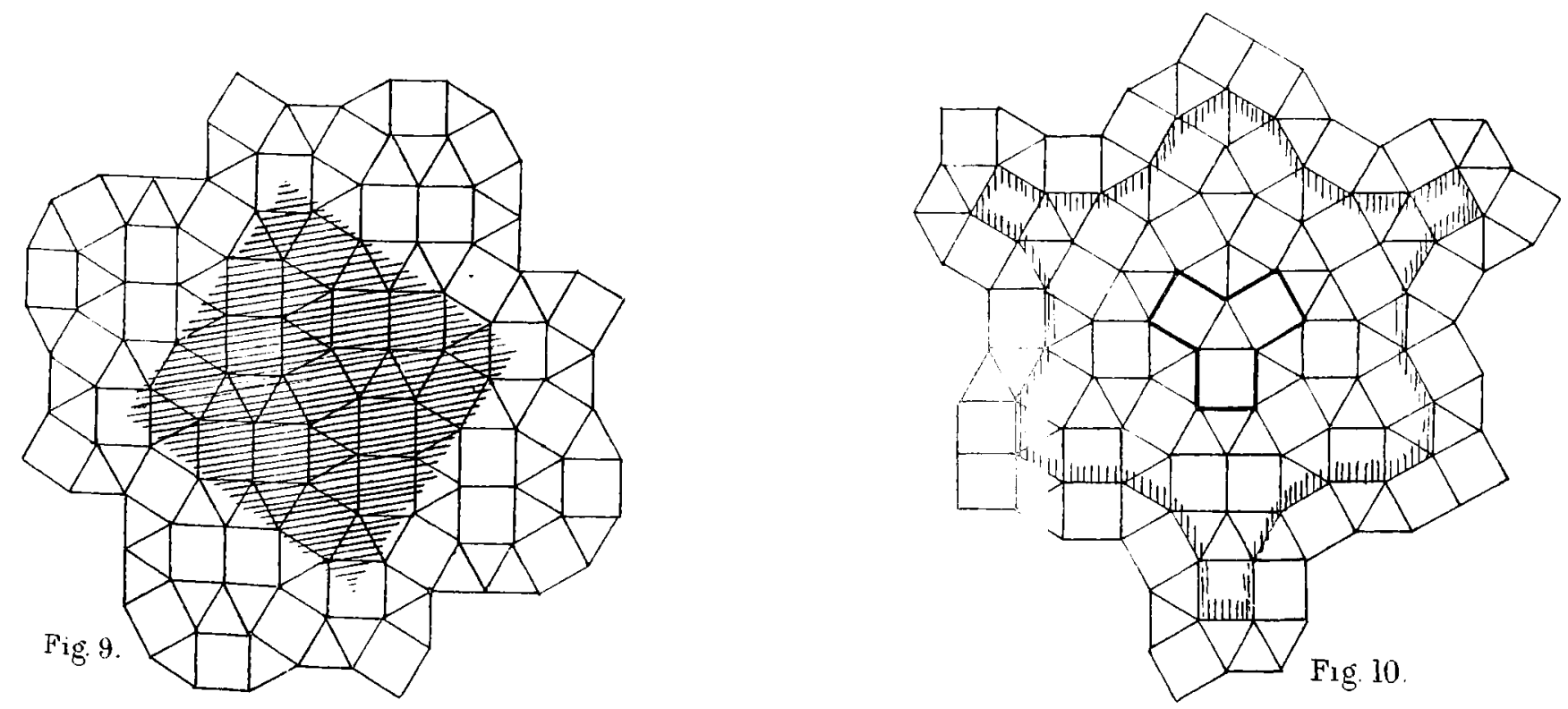
Trans. Roy. Soc. Edinr.

Vol. XL1.

Sommerville-Networks of the Plane in Absolute Geometry.-Plate IV.

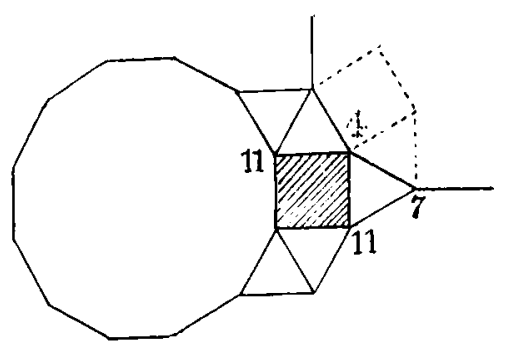

Fig. 1.

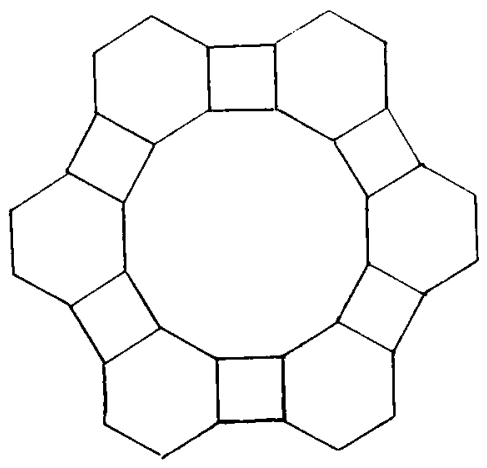

Fig. 13

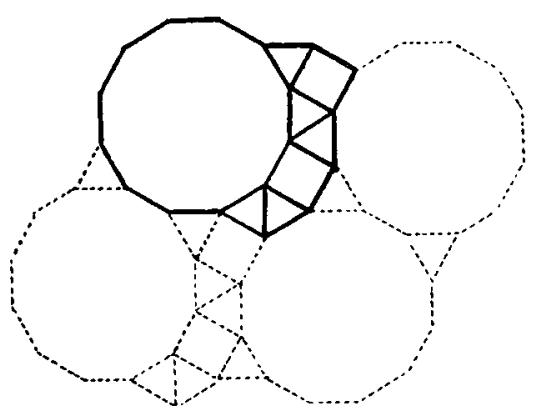

Fig. 15

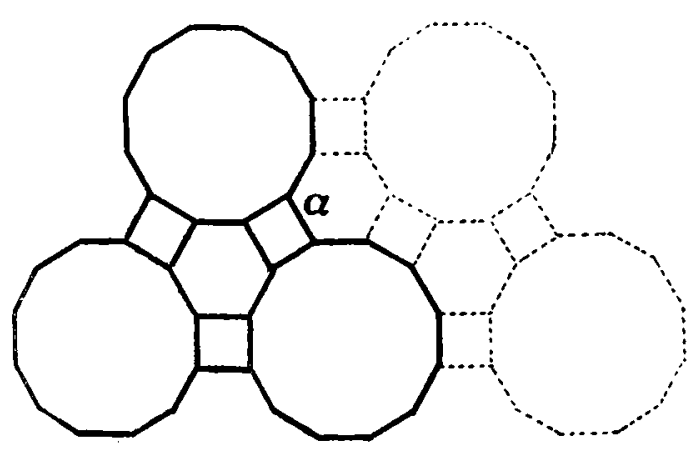

Fig. 17.

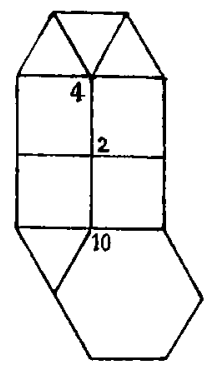

Fig. 11.

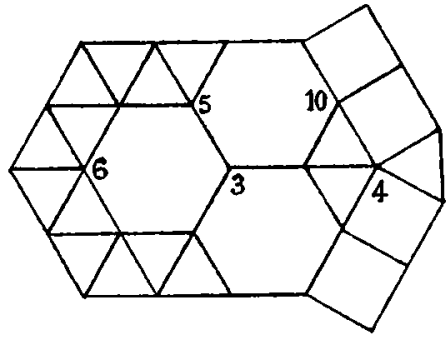

Fig. 12

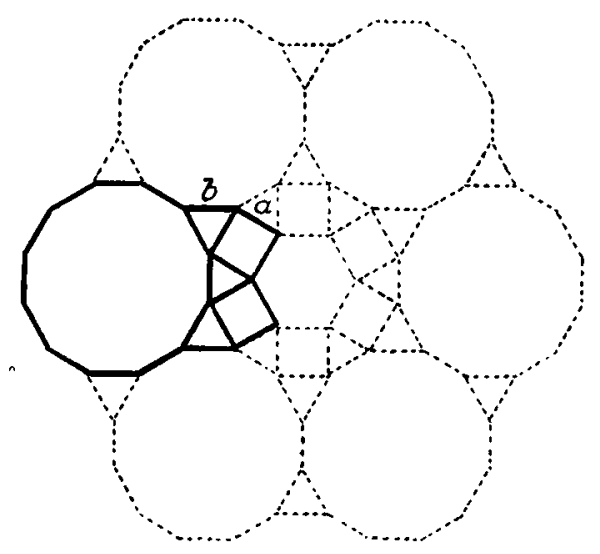

Fig. 14.

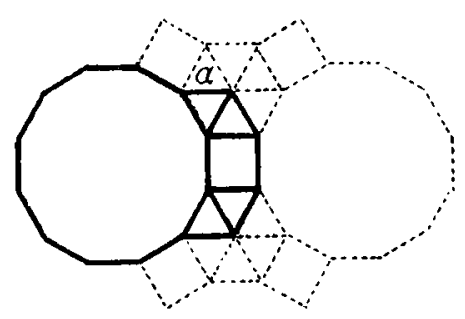

F 1 g. 16

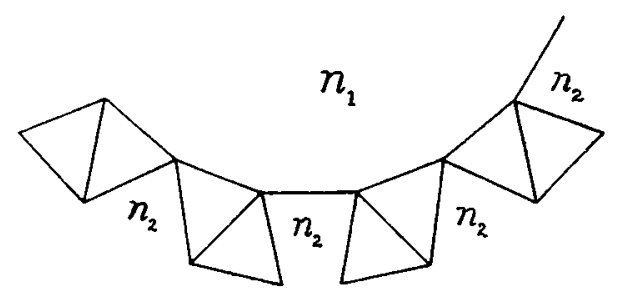

Fig. 18. 
Trans. Roy. Soc. Edinr.

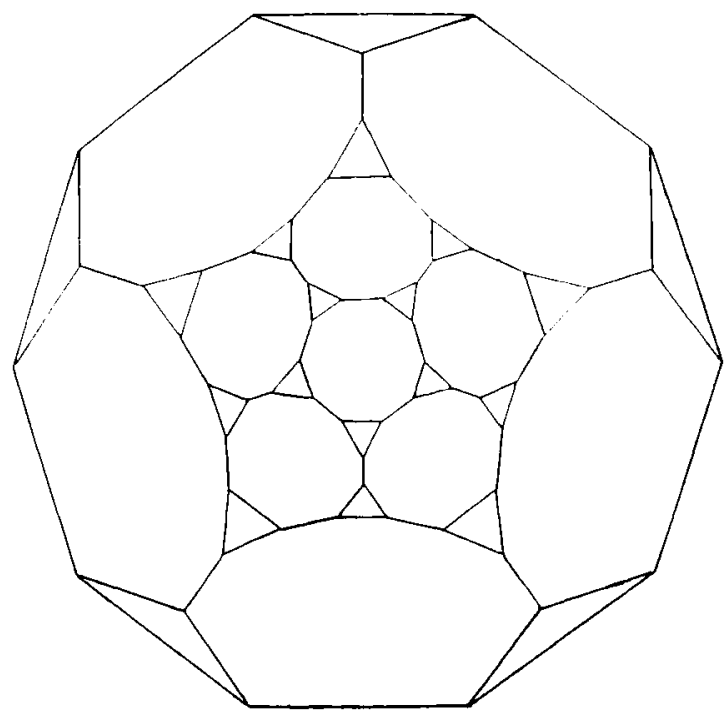

Fig. 19.

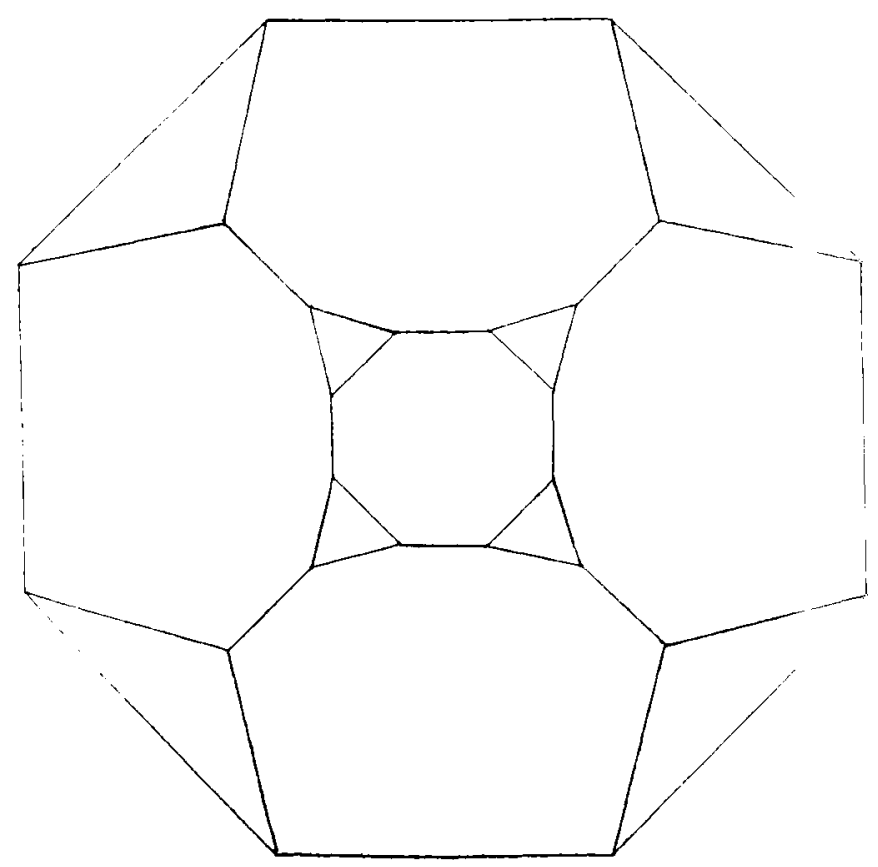

Fig. 20.

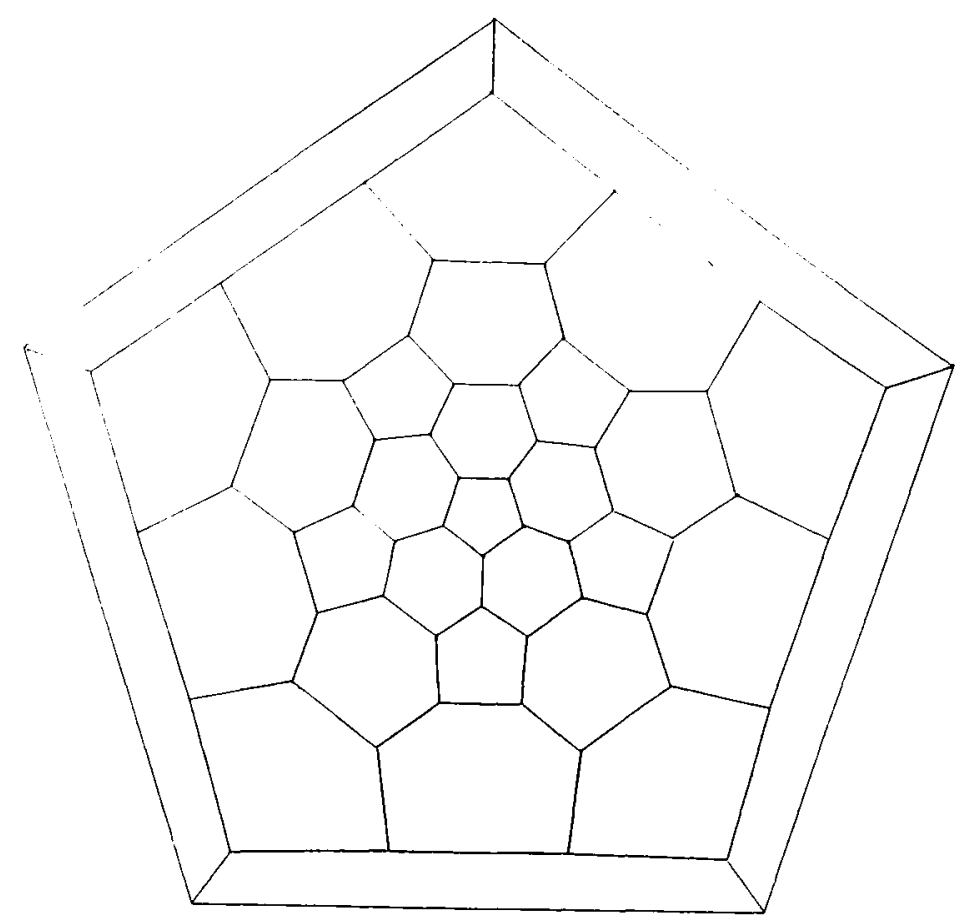

Fig. 21

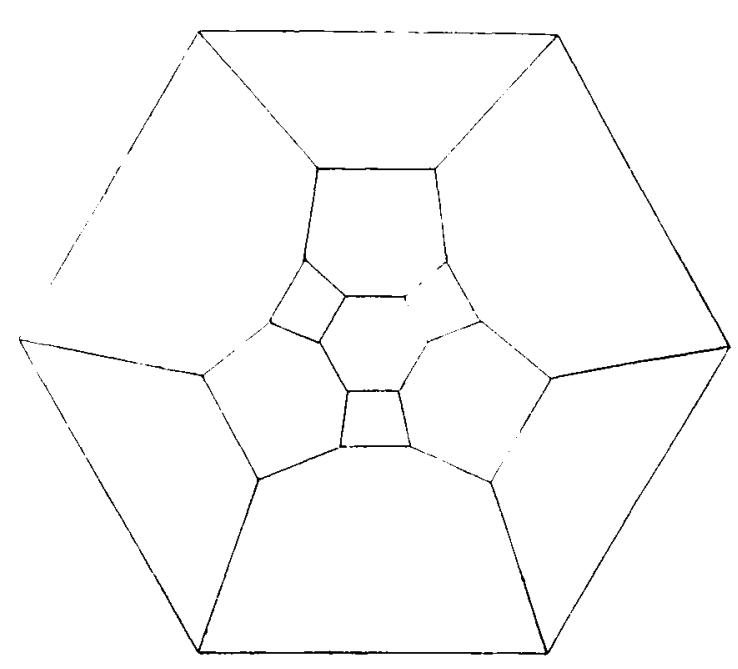

Fig. 22. 
Trans. Roy. Soc. Edinr.

Vol. XLI.

Sommervilif:- Networks of the Plane in Absolute Geometry.-Plate VI.
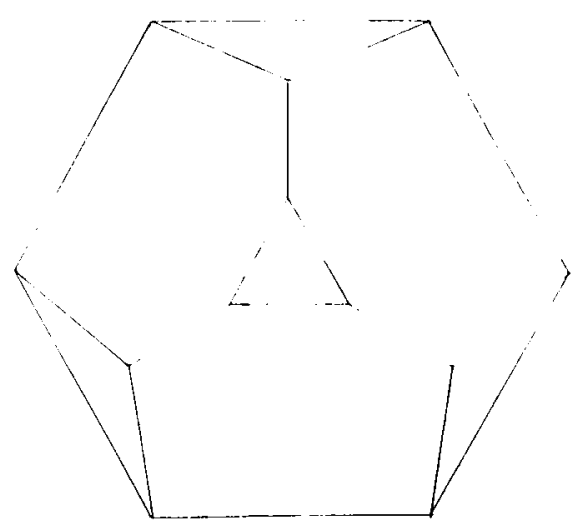

Fig. 23.

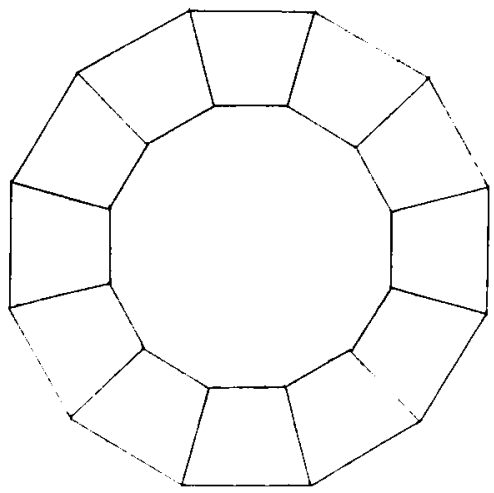

Fig. 24

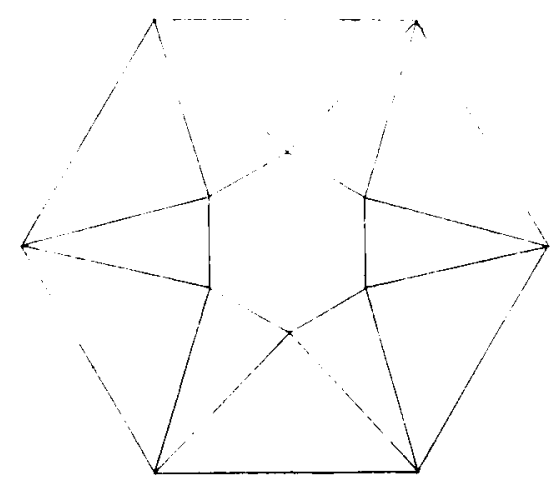

Fig. 25.

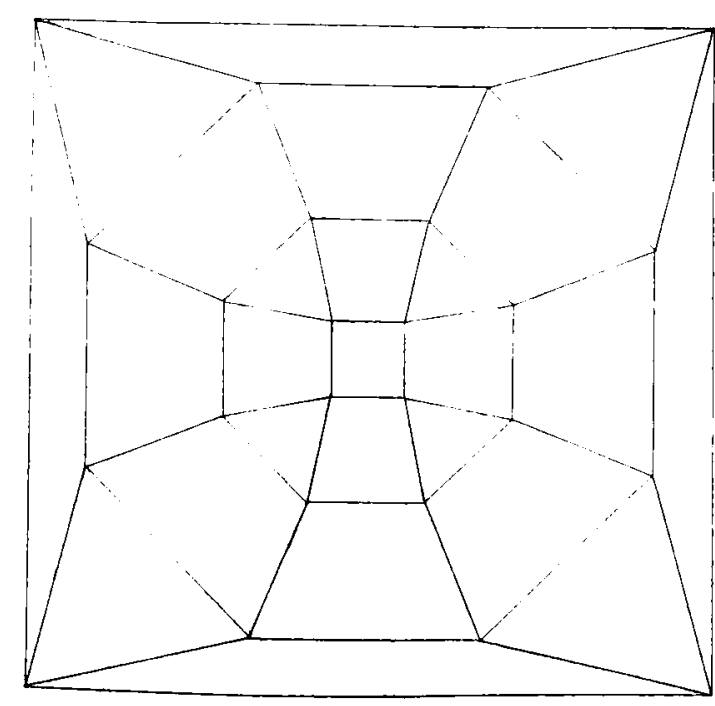

Fig. 26.

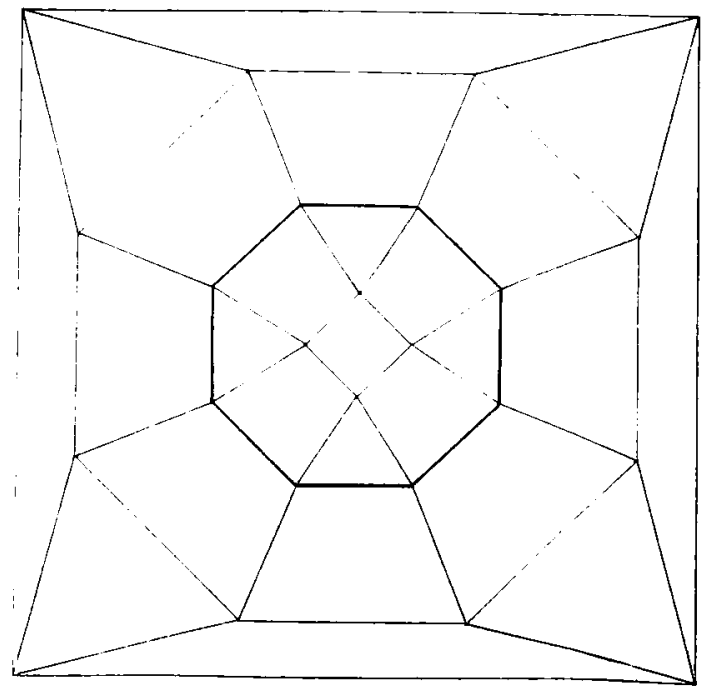

Fig. 27. 
Trans. Roy. Soc. Edin r.

Vol. XLI.

Sommerville--Networks or the Plane in Absolute Geometry.-Plate VII.

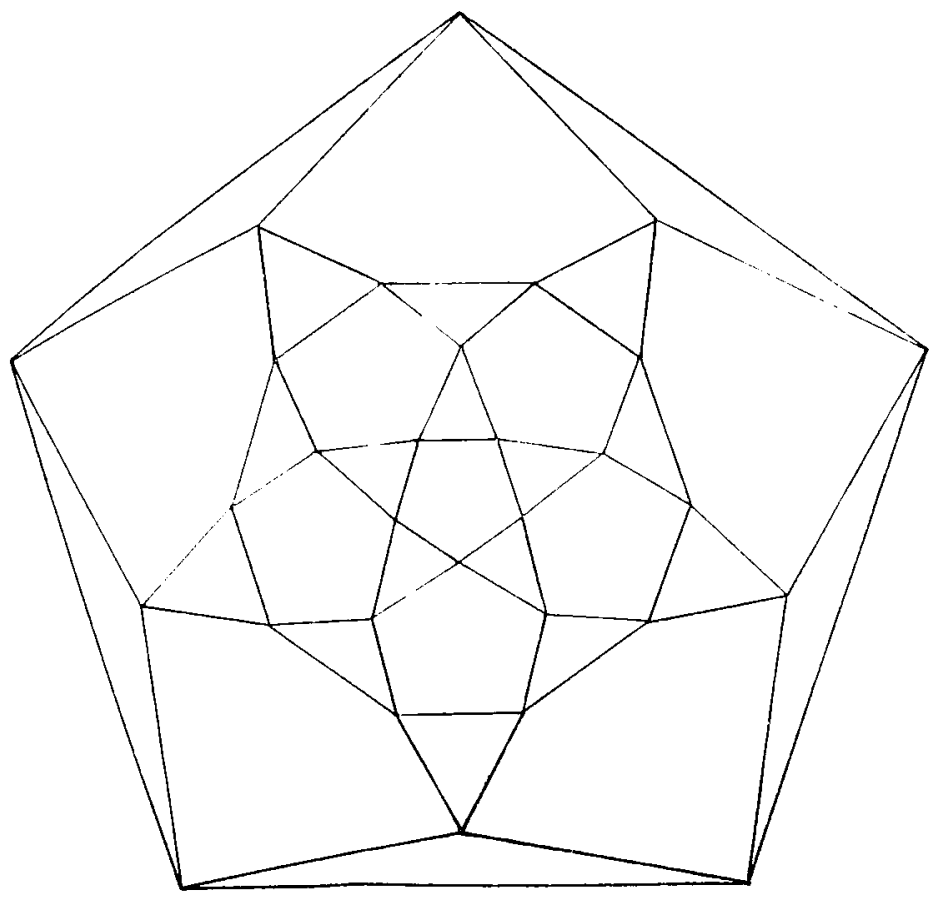

Fig. 28.

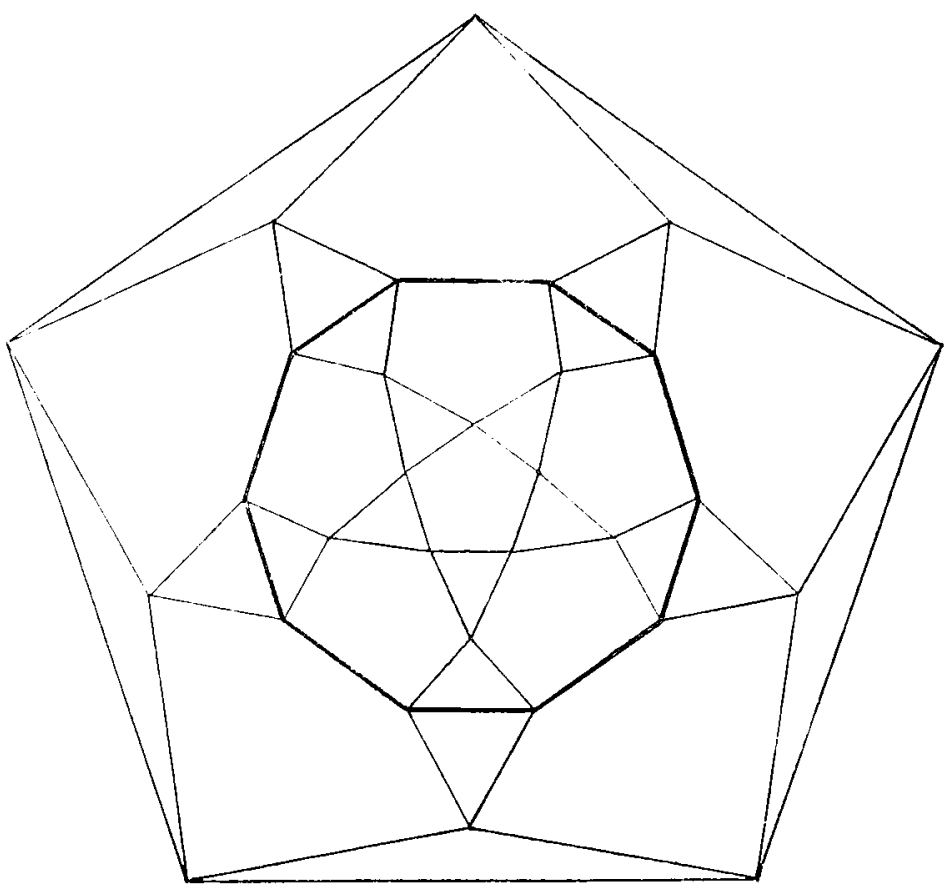

Fig. 29.

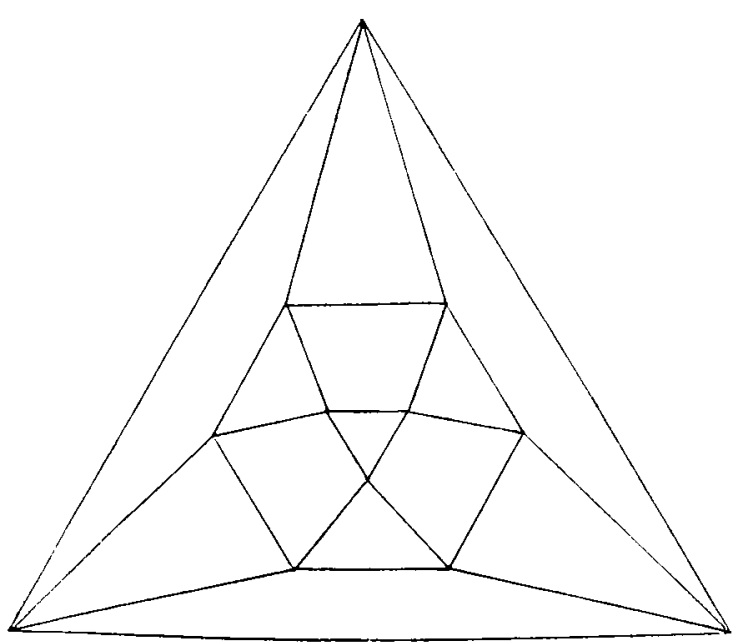

Fig. 30 .

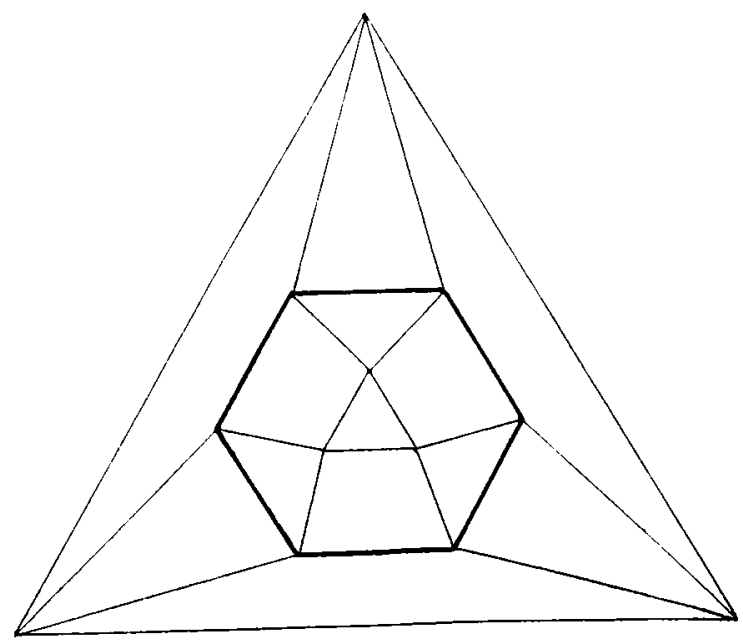

Fig. 31. 
Trans. Roy. Soc. Edin r.

Vol. XLI.

Sommerville-Networks of the Plane in Absolute Geometry.-Plate VIII

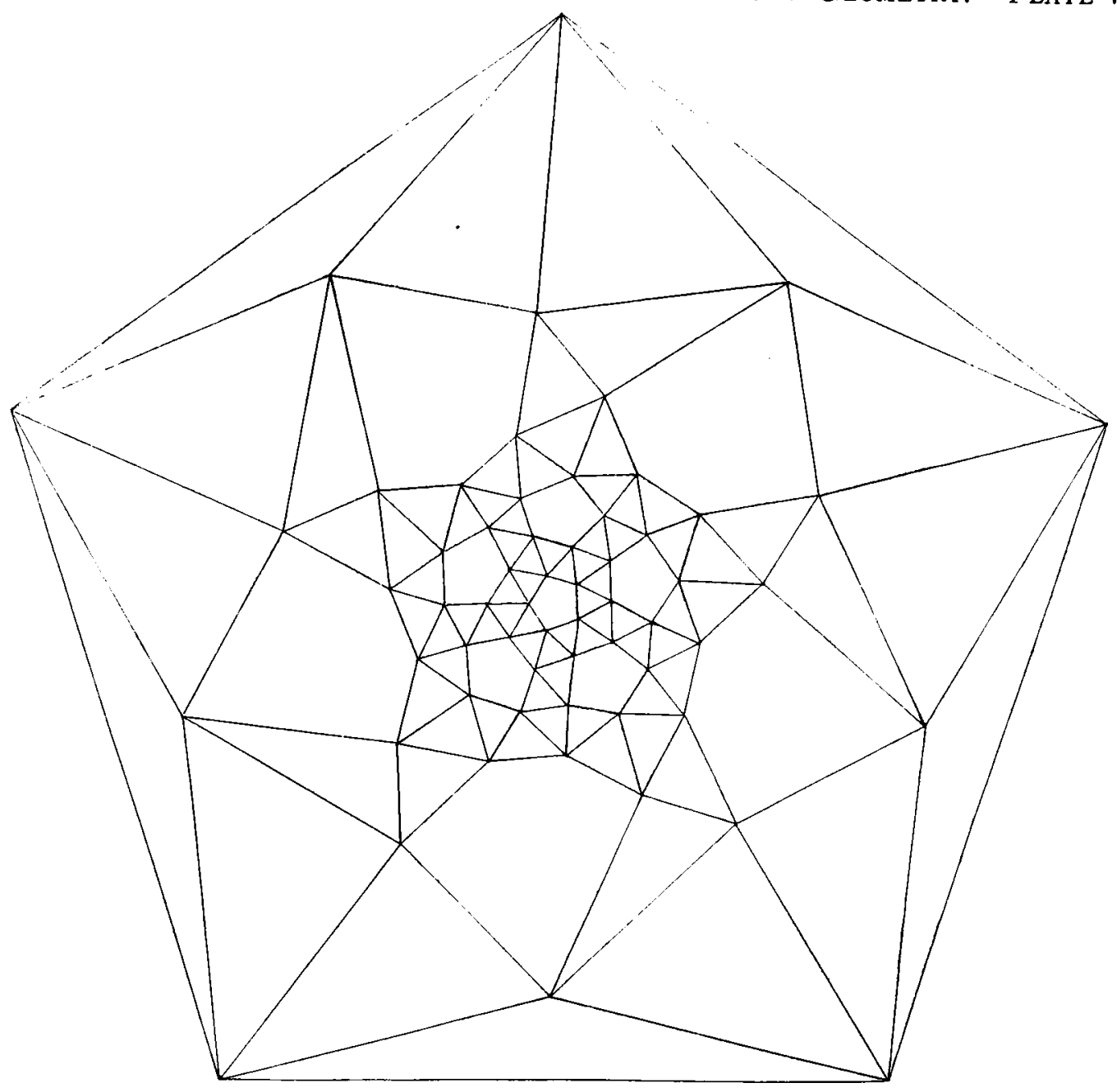

Fig. 32.

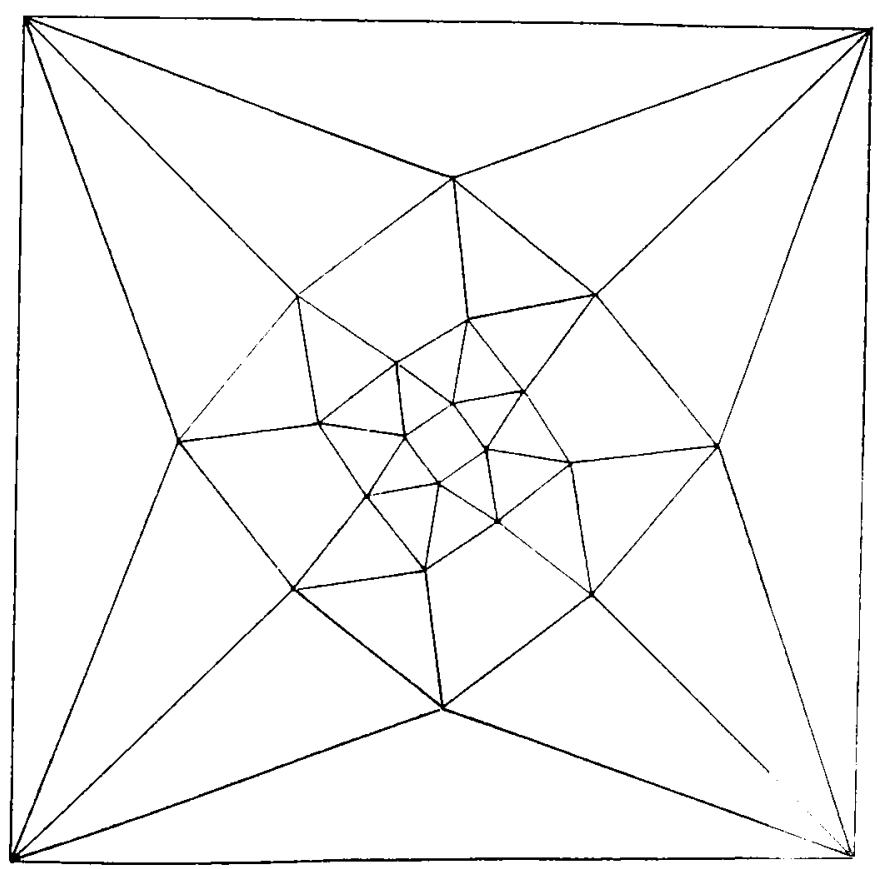

Fig. 33. 
Trans. Roy. Soc. Edin r.

Vol. XLI.

Sommerville-Networks of the Plane in Absolute Geometry.-Plate iX.

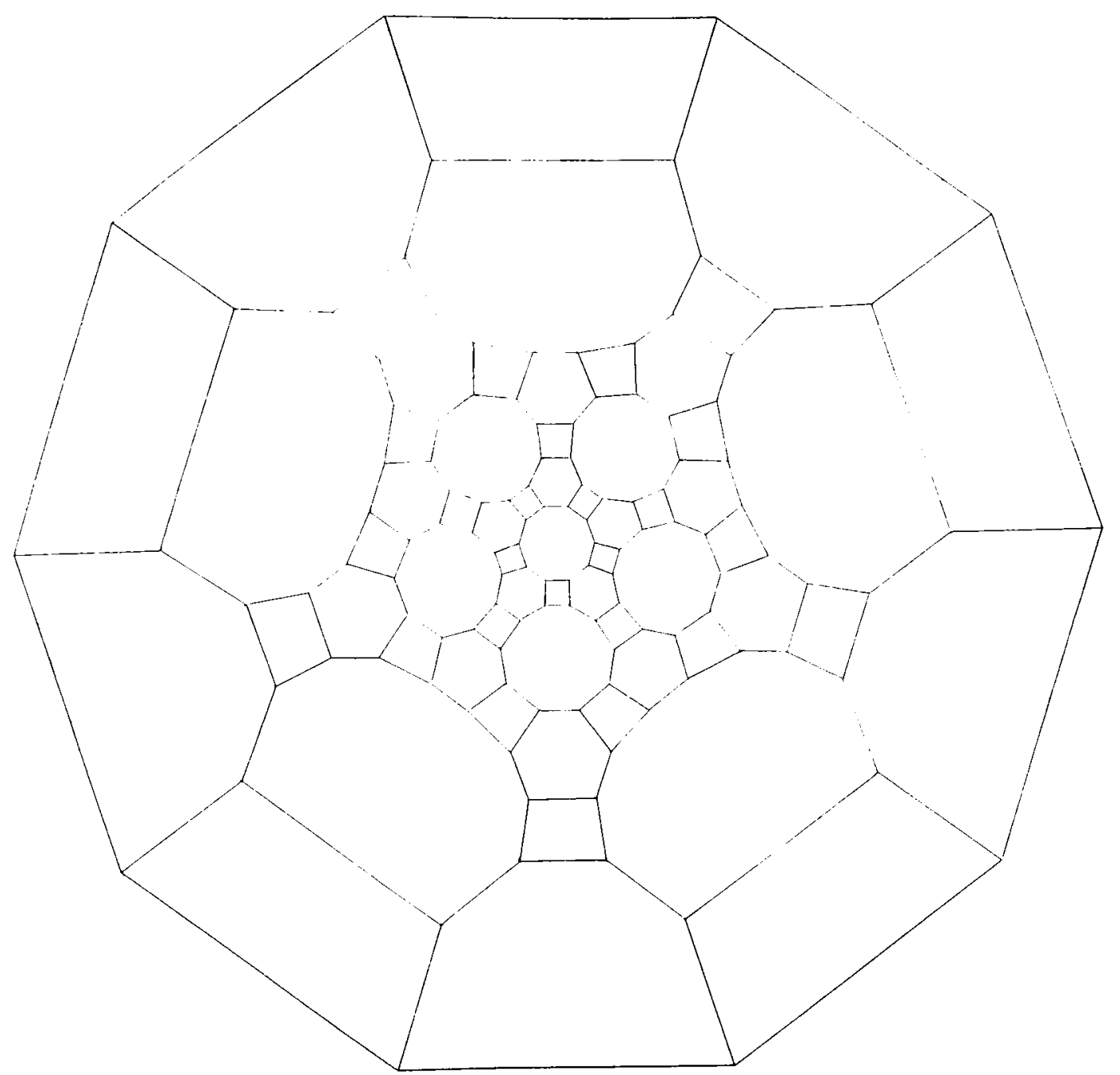

Fig. 34 .

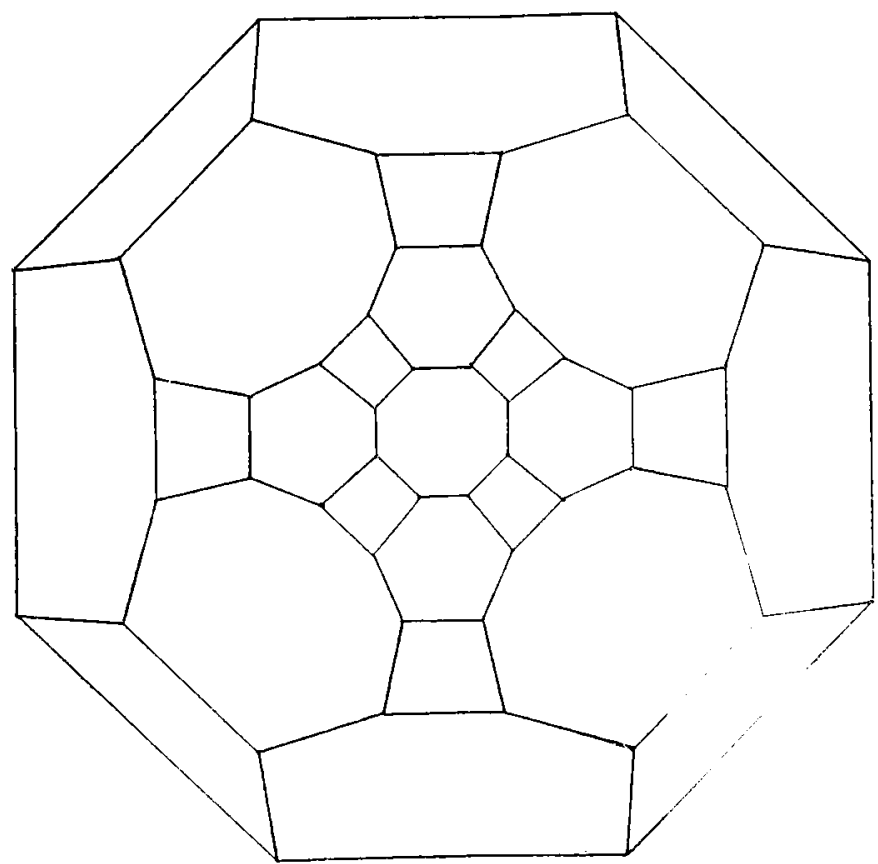

Fig. 35. 
Trans. Roy. Soc. Edin r.

Vol. XLI.

Sommervilie--Nitworks al: ine Planl: in Absolute Geometry.-Plate X.

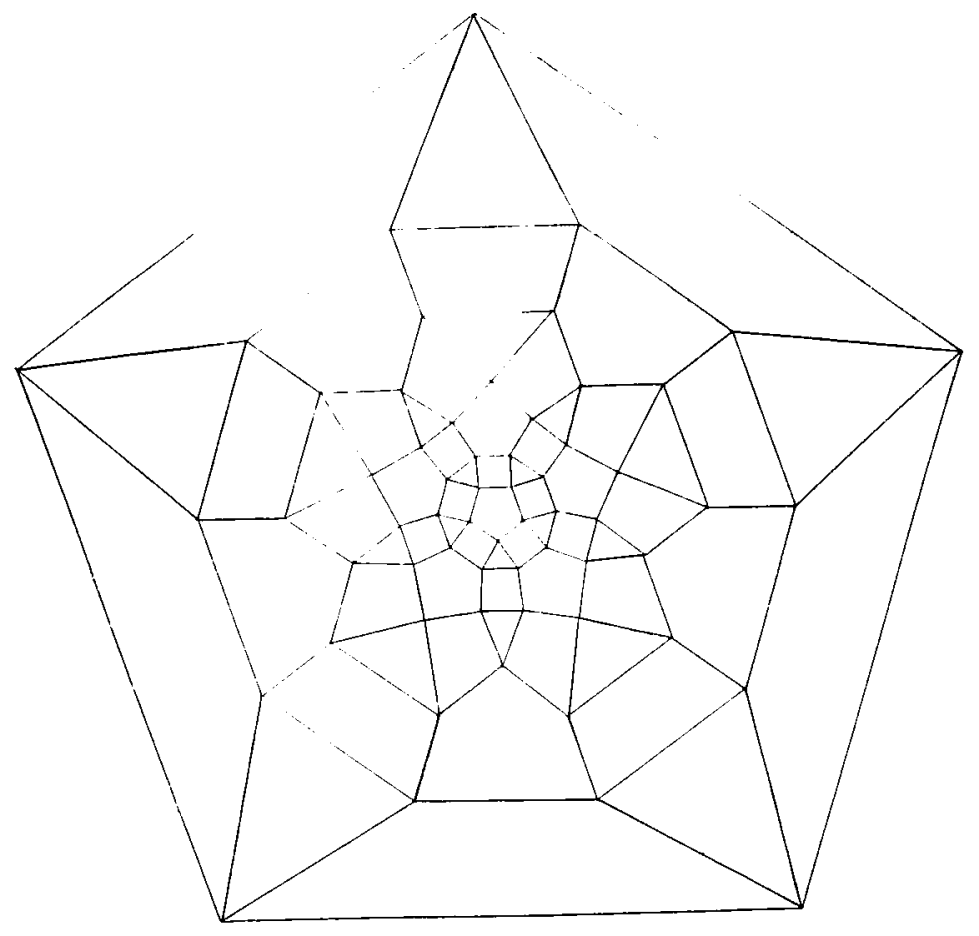

Fig. 36 .

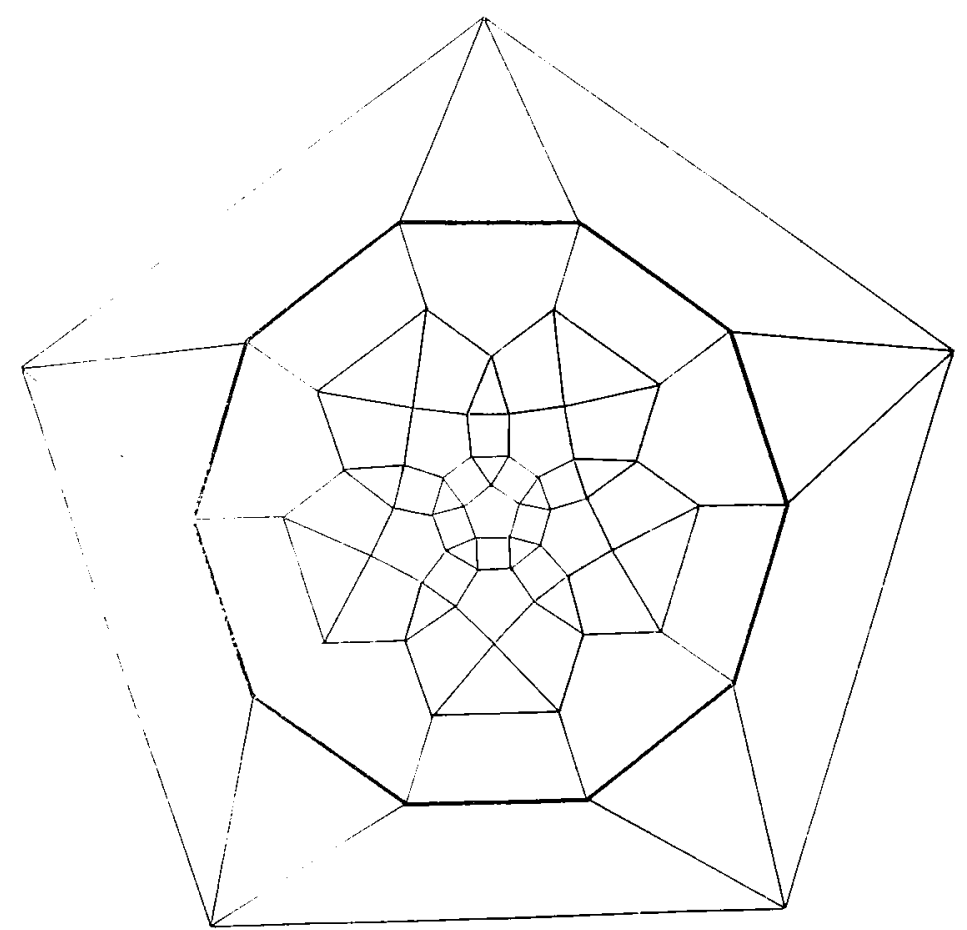

Fig. 37. 
Trans. Roy. Soc. Edinr.

Vol. XLI

Sommerville-Networks (IF the Plane in Absolute Geometry.-Plate XI.

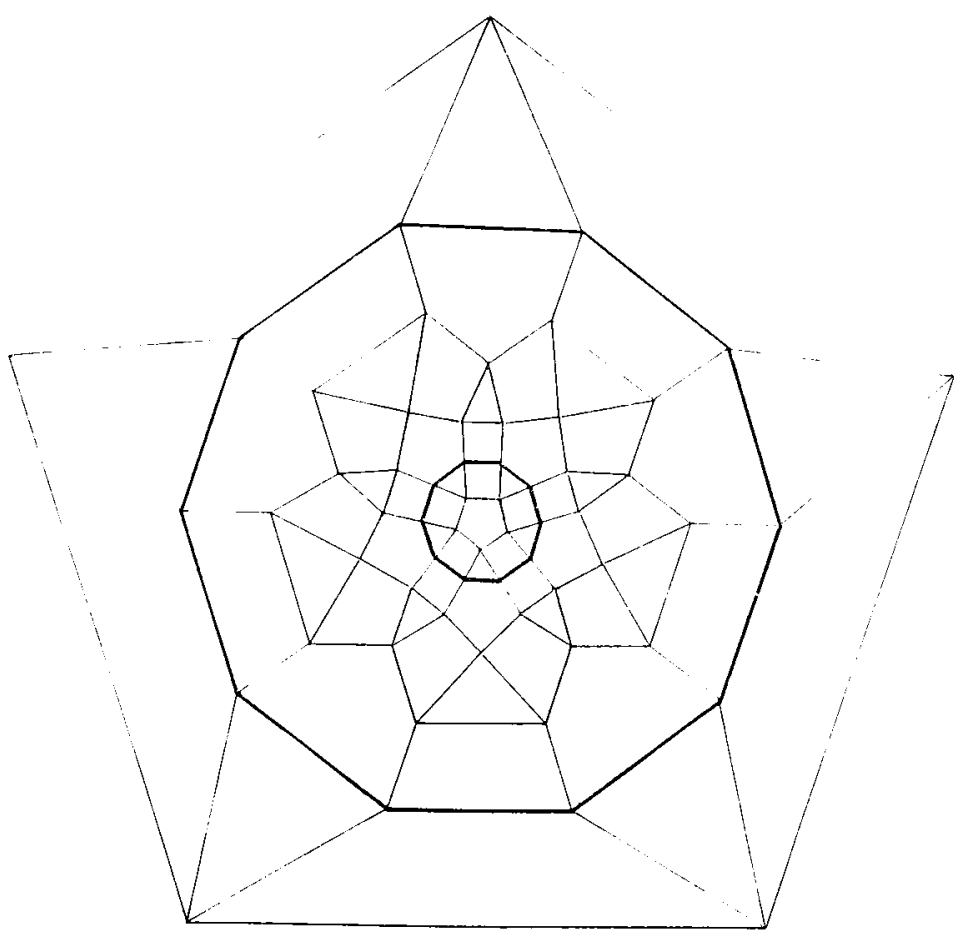

Fig. 38 .

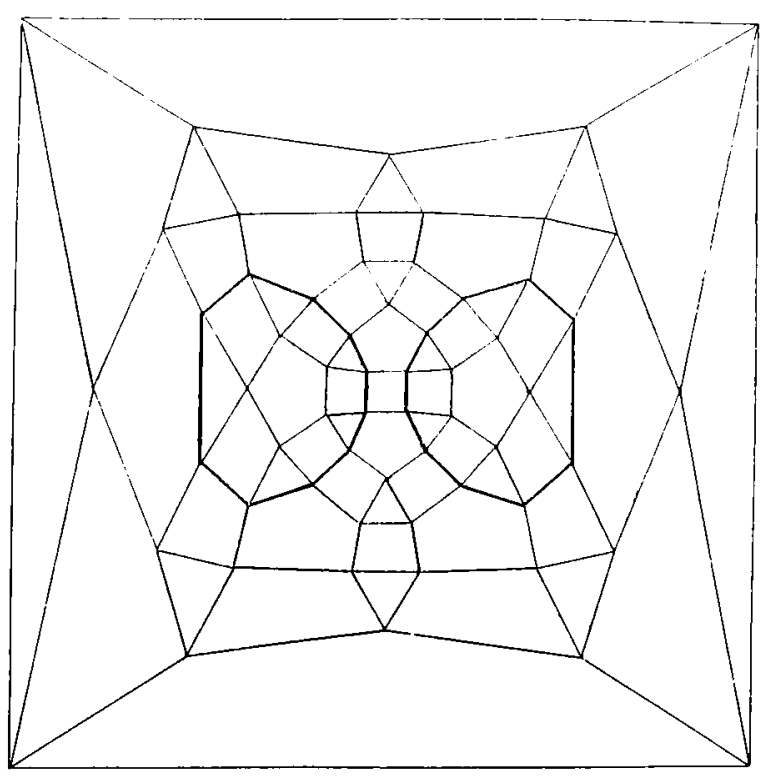

Fig. 39. 
Trans. Roy. Soc. Edin r.

Vol. XLI.

Sommerville: Nlitwoklis (h the Plane in Absolute Geometry.-Plate XII.

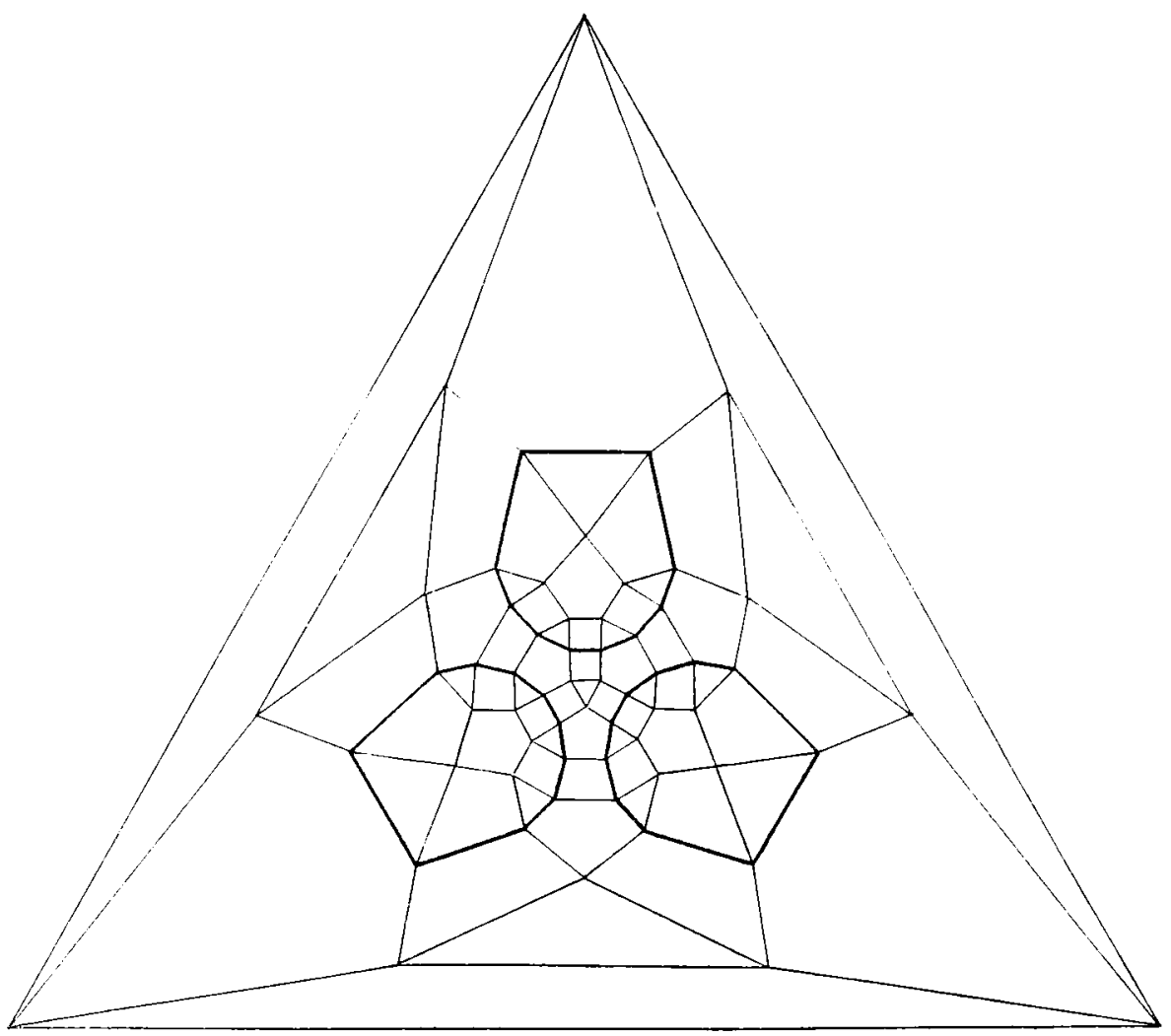

Fig. 40 .

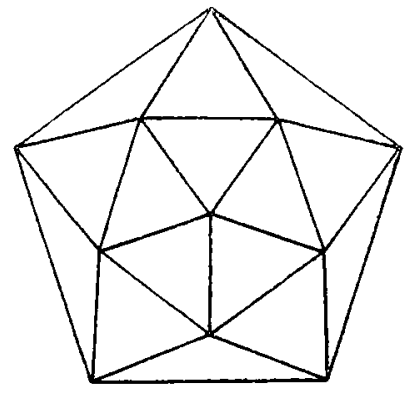

Fig. 41.

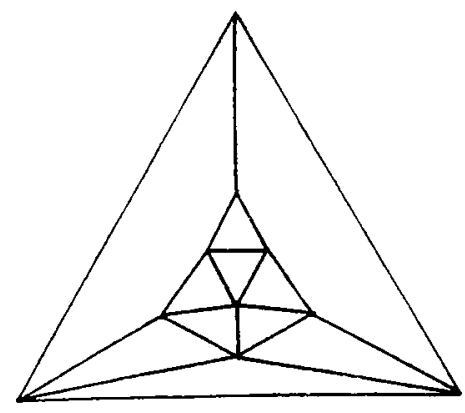

Fig. 42.

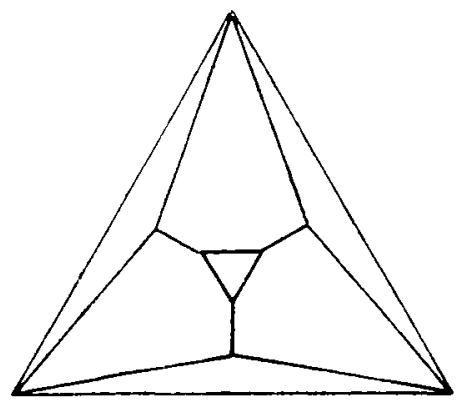

Fig. 43.

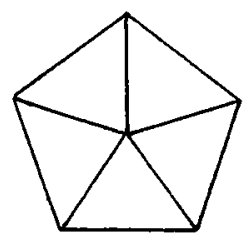

Fig. 44

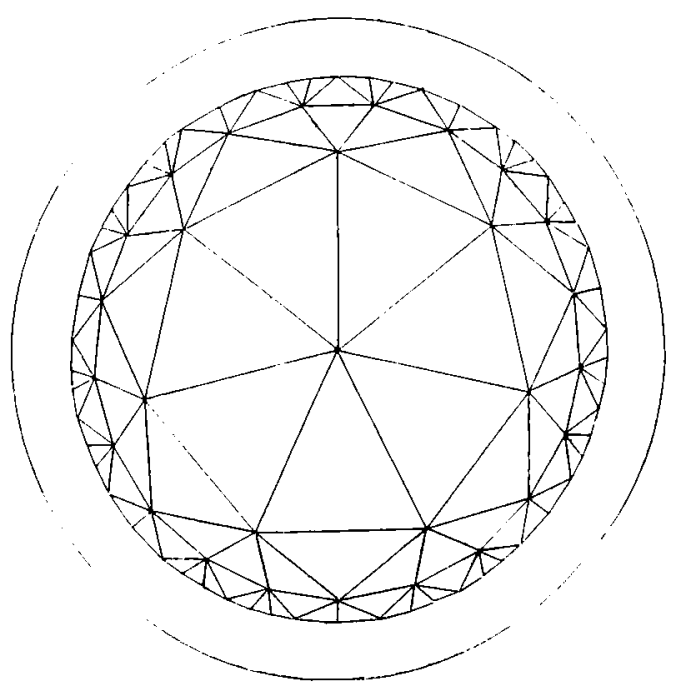

Fig. 46.

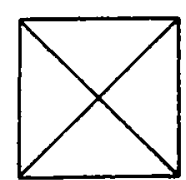

Fig. 45 . 